\title{
On a Hele-Shaw type domain evolution with convected surface energy density
}

\author{
M. GÜNTher ${ }^{1}$ AND G. ProkerT ${ }^{2}$ \\ ${ }^{1}$ Mathematisches Institut, Universität Leipzig, \\ Augustusplatz 10/11, 04109 Leipzig, Germany \\ email: Matthias.Guenther@math.uni-leipzig.de \\ 2 Department of Mathematics and Computing Science, Eindhoven, \\ University of Technology, PO Box 513, 5600 MB Eindhoven, The Netherlands \\ email: g.prokert@tue.nl
}

\begin{abstract}
Interest is directed to a moving boundary problem with a gradient flow structure which generalizes surface-tension driven Hele-Shaw flow to the case of nonconstant surface tension coefficient taken along with the liquid particles at the boundary. In the case with kinetic undercooling regularization well-posedness of the resulting evolution problem in Sobolev scales is proved, including cases in which the surface tension coefficient degenerates. The problem is reformulated as a vector-valued, degenerate parabolic Cauchy problem. To solve this, we prove and apply an abstract result on Galerkin approximations with variable bilinear forms.
\end{abstract}

Keywords: Free boundary motion, degenerate nonlocal parabolic evolution

MSC (1991): 35R35, 76B07

\section{Introduction}

Various experimental studies investigate the influence of spatial variations of the surface energy density (corresponding to the surface tension coefficient $\gamma$ ) on surface tension driven Hele-Shaw flows (cf. e.g. [14]). However, a mathematical model for such flows seems to be lacking. In this paper, a first step is attempted to close this gap. We derive and investigate a moving boundary problem (MBP) which arises, at least from a mathematical point of view, as a natural generalization from the case where $\gamma$ is a positive constant to the case of variable, nonnegative $\gamma$. Let us give an informal description of this generalization here; for the details we refer to Section 2 .

The Hele-Shaw MBP with constant $\gamma$ is well investigated. In particular, our starting point is the following observation [1,7]: On the Fréchet manifold $M$ of the surfaces $\Gamma$ that bound a domain of fixed given volume, an evolution $t \mapsto \Gamma_{t}$ satisfying the MBP can be interpreted as a gradient flow with respect to the energy functional

$$
\mathscr{E}=\mathscr{E}(\Gamma):=\gamma \operatorname{meas}(\Gamma)
$$


and the Riemannian metric $g$ given by (2.4).

In our generalization to nonconstant $\gamma$ we use the energy functional

$$
\mathscr{E}=\mathscr{E}(\Gamma, \gamma):=\int_{\Gamma} \gamma d \Gamma
$$

and keep the demand that the evolution is given by a gradient flow with respect to the same Riemannian metric. (A parallel procedure applied to viscous free boundary flows leads to the usual description of the Marangoni effect.) This leads to two related difficulties: First, the functional $\mathscr{E}$ is not longer depending on $\Gamma$ only. This is resolved in the following way: Instead of the manifold $M$ we consider the vector bundle $F$ over $M$, having as fiber space at $\Gamma$ the (smooth) functions on $\Gamma$. On this bundle, $\mathscr{E}$ is well defined. Secondly, one also has to prescribe an evolution law for $\gamma$ as a function on the moving surface $t \mapsto \Gamma_{t}$. Again, we make a simple choice: We assume $\gamma$ to be transported along with the velocity field at the boundary, and we allow the tangential transport to be diminished by a "slip factor" $\delta \in[0,1]$. The case $\delta=1$ describes a fixed coupling of the values of $\gamma$ to the moving liquid particles. Physically, this would occur e.g. if $\gamma$ is temperature dependent and heat conduction is negligible. On the other hand, the case $\delta=0$ corresponds to transport in normal direction only. In differential geometric terms, this transport law is realized by introducing a suitable connection $D$ on $F$ and demanding parallel transport of $\gamma$, see (2.7)-(2.10).

Let us remark here that we do not claim that these assumptions are necessarily in accordance with the physics of an actual Hele-Shaw flow with nonuniform surface energy density, e.g. induced by the presence of a surfactant. It is well conceivable that the interface dynamics in such a situation might be dominated by more complex phenomena like the occurrence of boundary layers, thin surfactant films, or other effects. For instance, if a surfactant is present, one has to solve a transport equation for the surfactant concentration and to determine $\gamma$ from this. (See [16] for the case of Stokes flow; such a modification of our problem would not present new principal difficulties.)

To test our assumptions in a concrete situation, numerical work as well as comparison with experiments would be necessary. However, even our simple model is of mathematical interest in its own right and as a typical example for nonlocal, degenerate parabolic evolutions.

In Section 2 of this paper we derive the moving boundary problem (2.12), (2.13) from the gradient flow formulation. In the sequel, we prove our main result, namely, a local existence and uniqueness result for this problem in scales of Sobolev spaces. For the precise formulation and further results concerning continuous dependence on the initial data see Theorems 3.1 and 3.2 below. If the surface $\Gamma_{t}$ and the coefficient $\gamma_{t}$ are known at some time $t$, then the velocity potential $\phi_{t}$ is completely determined by (2.12). If one parametrizes $\Gamma_{t}$ over a fixed reference surface $S$, the moving boundary problem can be interpreted as an evolution equation with nonlinear, nonlocal pseudodifferential operators. The parametrization can be constructed in at least two different ways: On one hand, it is possible to parametrize the surfaces using one scalar function, e.g. the normal distance to the reference surface. Then $\gamma_{t}$ has to satisfy a transport equation whose coefficients depend on the parametrization and on the velocity potential. On the other hand, the moving boundary can also be represented by mappings $u(\cdot, t): S \rightarrow \mathbb{R}^{m}$ 
whose time derivatives are given by the velocity vector, i.e.

$$
\partial_{t} u=\mathscr{F}(u):=\left(\left(\nabla_{N}+\delta \nabla_{T}\right) \phi_{t}\right) \circ u,
$$

where $\nabla_{N}$ and $\nabla_{T}$ denote the normal and tangential component of the gradient, respectively.

This formulation, which we will use in the sequel, is $\mathbb{R}^{m}$-valued. Therefore, the corresponding Cauchy problem will be necessarily degenerate, even if $\gamma$ is strictly positive (or even constant). However, this is no crucial disadvantage as our problem couples a transport equation with a parabolic evolution and we allow $\gamma$ to degenerate as well. Our approach has two favorable properties: $\gamma$ now appears only as a known, time-independent function on the reference domain, and the additional freedom in the choice of the diffeomorphisms can be used to derive generalized chain rules for our nonlocal operators which reduce the technical effort in the proofs of the necessary estimates.

As long as $\gamma$ is nonnegative, the normal component of the linearisation $\mathscr{F}^{\prime}(u) v$ behaves as a degenerate elliptic second order operator on the normal component of $v$, so that we have, e.g., with respect to the $L^{2}$-inner product

$$
\left\langle n \cdot v, n \cdot \mathscr{F}^{\prime}(u) v\right\rangle_{L^{2}} \leq C\|v\|_{L^{2}}^{2} .
$$

An estimate like this does not hold for the complete linearization, including the tangential components. Due to the special structure of $\mathscr{F}$, however, it is possible to define inner products $\langle,\rangle_{u}$ which define equivalent norms on $L^{2}$ and satisfy

$$
\left\langle v, \mathscr{F}^{\prime}(u) v\right\rangle_{u} \leq C\|v\|_{L^{2}}^{2} .
$$

Defining higher order inner products $\langle,\rangle_{u, s}$ on the basis of $\langle\cdot, \cdot\rangle_{u}$, one finally can show an $H^{s}$-energy estimate

$$
\langle\mathscr{F}(u), u\rangle_{u, s} \leq C\|u\|_{s}^{2}
$$

for $s$ sufficiently large, and, on the other hand, the dependence of these inner products on $u$ can be controlled by a weaker Sobolev norm. In a suitable abstract functional analytic framework, these estimates can be used to obtain proofs for our main results. Moreover, these results implies the existence of an unique solution $w:=(\operatorname{Id}-\lambda \mathscr{F})^{-1}$ of the equation $w-\lambda \mathscr{F}(w)=v$ provided $\lambda \geq 0$ sufficiently small. The solution of the Cauchy problem for the evolution equation is given by the exponential formula

$$
u(t)=\lim _{n \rightarrow \infty}\left(\operatorname{Id}-\frac{t}{n} \mathscr{F}\right)^{-n} u(0)
$$

with convergence in $H^{s}$ provided $u(0) \in H^{s}, s$ sufficiently large.

The structure of the paper after Section 2 is as follows: In Section 3, we introduce the necessary notation and announce our main results together with the abstract existence theorems which are used. Section 4 is devoted to the behavior of our (nonlocal) operators in scales of Sobolev spaces, and in Section 5 the $u$-dependent inner products are introduced, and the necessary estimates are shown. Finally, the main results (Theorems 3.1 and 3.2) are proved in Section 6. The proof of a general abstract existence result (Theorem 3.4), which may be of independent interest, is given in the Appendix. 


\section{The equations of motion}

Here we characterize the moving boundary problem as abstract gradient flow on the manifold of the natural configuration space. We start by recalling the following general properties of incompressible, source free Hele-Shaw flows. One looks for a family of domains $\Omega(t) \subset \mathbb{R}^{m}$ parametrized by time $t \geq 0$ and corresponding velocity fields $\mathbf{v}(\cdot, t)$ such that (according to Darcy's law)

$$
\mathbf{v}(\cdot, t)=\nabla \varphi(\cdot, t) \text { in } \Omega(t)
$$

with a potential field $\varphi(\cdot, t)$ proportional to negative pressure. As we also demand that the boundary $\Gamma(t)$ of $\Omega(t)$ moves along with the velocity field, we find the kinematic boundary condition

$$
V_{n}(t)=\partial_{n} \varphi(\cdot, t) \text { on } \Gamma(t),
$$

where $V_{n}(t)$ is the normal velocity of the moving boundary $\Gamma(t)$ and $\partial_{n}=\partial / \partial n$ is the derivative in direction of the unit outward normal $n(t)$ of $\Gamma(t)$. As $\mathbf{v}$ is divergence-free,

$$
\Delta \varphi(\cdot, t)=0 \text { in } \Omega(t) .
$$

Thus, in any Hele-Shaw flow, the complete velocity field is determined by the normal velocity at the boundary.

If the surface tension coefficient is a positive constant, the corresponding surface energy is proportional to the surface area and the Hele-Shaw flow driven by surface tension can be interpreted as abstract gradient flow of this functional with respect to an appropriately chosen inner product, cf. $[1,7]$. As this formulation is a main ingredient in our derivation of the moving boundary problem below, we define this inner product more precisely. Consider for the time being a fixed smooth domain $\Omega$ with boundary $\Gamma$ and define

$$
V_{\Gamma}:=\left\{v \in C^{\infty}(\Gamma) \mid \int_{\Gamma} v d \Gamma=0\right\} .
$$

The space $V_{\Gamma}$ can be interpreted as space of all possible normal boundary velocities, the restriction expresses conservation of volume. We fix $\beta \geq 0$ and introduce on $V_{\Gamma}$ the bilinear form $g_{\Gamma}$ given by

$$
g_{\Gamma}\left(v_{1}, v_{2}\right):=\int_{\Omega} \nabla \varphi_{1} \nabla \varphi_{2} d x+\beta \int_{\Gamma} v_{1} v_{2} d \Gamma
$$

where the $\varphi_{i}, i=1,2$ are (weak) solutions of the Neumann problems

$$
\Delta \varphi_{i}=0 \text { in } \Omega, \quad \partial_{n} \varphi_{i}=v_{i} \text { on } \Gamma .
$$

To give a physical interpretation of the quadratic functional $v \mapsto g_{\Gamma}(v, v)$ we remark that the first term represents energy dissipation by the corresponding Hele-Shaw flow (cf. [7]) while for $\beta>0$ the second term is a penalty for large normal boundary velocities. Note that, by Green's formula,

$$
g_{\Gamma}\left(v_{1}, v_{2}\right)=\int_{\Gamma}\left(\varphi_{1}+\beta \partial_{n} \varphi_{1}\right) v_{2} d \Gamma .
$$

In differential geometric terms, this inner product arises a Riemannian metric on the Fréchet manifold $M$ of boundaries $\Gamma=\partial \Omega$ to smooth compact domains $\Omega \subset \mathbb{R}^{m}$ with given fixed volume. By interpreting a tangent vector at $\Gamma \in M$ as normal velocity field 
of the boundary, there is a natural way of thinking of vector fields $X$ on $M$ as sections in the Fréchet vector bundle $E=\cup_{\Gamma \in M} V_{\Gamma}$ with base $M$ and fiber $V_{\Gamma}$, i.e. there is a natural isomorphismus $T_{\Gamma} M \simeq V_{\Gamma}$ and we have for any real functional $J \in C^{\infty}(M)$

$$
(X J)(\Gamma)=\left.\partial_{\varepsilon} J\left(\Gamma_{\varepsilon}\right)\right|_{\varepsilon=0},
$$

where $\varepsilon \mapsto \Gamma_{\varepsilon} \in M$ is a path of admissibles shapes with normal velocity $v$ for $\varepsilon=0$,

$$
\Gamma_{\varepsilon}:=\left\{x_{\varepsilon} \mid x \in \Gamma\right\}, \quad x_{\varepsilon}:=x+\varepsilon(v(x)+O(\varepsilon)) n(x) .
$$

Thus, identifying a vector $X_{\Gamma} \in T_{\Gamma} M$ in this sense with its image $v \in V_{\Gamma}$ and considering smooth domain dependence of the solution to a Neumann problem, $\Gamma \mapsto g_{\Gamma}$ defines a Riemannian metric $g$ on the manifold $M$. It is remarkable that in the case $\beta=0$ a geodetic line w.r. to this metric $g$ represents the motion of an incompressible irrotational perfect fluid with a free boundary; for the corresponding Levi-Civita derivative, Riemannian curvature and an analysis of the Jacobi equation from a differential geometric point of view we refer to [3].

Now, considering first the surface energy (1.1) with constant $\gamma$, the normal velocity $V_{n} \in V_{\Gamma}$ of a surface tension driven Hele-Shaw flow is determined by

$$
g_{\Gamma}\left(V_{n}, v\right)=-\mathscr{E}^{\prime}(\Gamma)\{v\} \text { for all } v \in V_{\Gamma},
$$

where $\mathscr{E}^{\prime}(\Gamma)\{v\}:=(X \mathscr{E})(\Gamma)$ denotes the derivative of the energy in direction of $X_{\Gamma} \simeq v$. As a consequence, at each instant of time $t$ the flow reduces the surface energy as rapidly as possible among all normal velocities with prescribed norm corresponding to the inner product (2.4), in particular, the flow is volume preserving and surface area decreasing. By a well-known formula for the first variation of surface area we find

$$
\mathscr{E}^{\prime}(\Gamma)\{v\}=-\int_{\Gamma} \kappa v d \Gamma
$$

where $\kappa$ is the mean curvature of $\Gamma$ with sign determined by the above variation formula (negative sign if $\Omega$ is convex); for notational convenience, throughout the paper the usual normalization of $\kappa$ has been changed by a cofactor $m-1$. To model the influence of a variable surface tension coefficient which is coupled on a transport mechanism, it arises now quite natural to consider a surface energy functional of the form (1.2) where $\gamma \geq 0$ denotes an surfaces energy density function along $\Gamma$, not necessarily constant. It should be noted that we don't assume a priori an one-to-one correspondence between the surface $\Gamma$ and density $\gamma$, as it is the case in simpler situations, e.g. where a known global function generates the density via restriction or where an anisotropic surface energy density is considered, i.e.

$$
\gamma=\left.f\right|_{\Gamma} \quad \text { or } \quad \gamma=f \circ n \text { on } \Gamma
$$

with given $f \in C^{\infty}\left(\mathbb{R}^{m}\right)$; the latter energy density is commonly used to model crystal growth problems. In fact, in our setting the functional $\mathscr{E}$ is uniquely defined on the vector bundle $F:=\cup_{\Gamma \in M} C^{\infty}(\Gamma)$ with base $M$ only. In such a case, computation of the derivative of the surface energy in direction of a given vector field requires a law for the change of $\gamma$ on the moving surface. Using differential geometric terms we make the following assumption: along a path $c$ in $M$ the energy density is transported by parallel 
displacement with respect to a given connection $D_{X}$ which acts on sections $\gamma$ in $F$, i.e.

$$
D_{\dot{c}} \gamma=0 \text { along } c .
$$

In further considerations we restrict our attention to the connection $D_{X}$ which is defined as follows: Let $X$ be any vector field, $\Gamma \in M$ and $v \in V_{\Gamma}$ with $X_{\Gamma} \simeq v$, then we set for any section $\gamma$ in $F$

$$
\left.D_{X} \gamma\right|_{\Gamma}:=\left.\partial_{\varepsilon} \bar{\gamma}_{\varepsilon}\right|_{\varepsilon=0}+\delta \nabla_{\Gamma} \psi \nabla_{\Gamma} \gamma, \quad \delta \in[0,1],
$$

where in terms of the notation $(2.6)$

$$
\bar{\gamma}_{\varepsilon}(x):=\bar{\gamma}_{\Gamma_{\varepsilon}}\left(x_{\varepsilon}\right), \quad(\varepsilon, x) \in\left(-\varepsilon_{0}, \varepsilon_{0}\right) \times \Gamma,
$$

and $\psi$ is a solution of the Neumann problem

$$
\Delta \psi=0 \text { in } \Omega, \quad \partial_{n} \psi=v \text { on } \Gamma .
$$

Interpretation of $D_{X}$ and parallel transport w.r. to $D_{X}$ is quite obvious in terms of the underlying Hele-Shaw flow. In contrast to the case of constant $\gamma$, we also have to consider the influence of the tangential motion at the boundary which results from a normal variation of the boundary. As pointed out before, in a Hele-Shaw flow the velocity field corresponding to a normal boundary velocity $v \in V_{\Gamma}$ is $\nabla \psi$ where $\psi$ solves (2.10). Hence, in the case $\delta=1,(2.7),(2.8)$ express that the surface energy density is transported along with the liquid particles, i.e. with the velocity field $\nabla \psi$ at the boundary. On the other hand, in case of $\delta=0$, transport in normal direction without any tangential movement is expressed. The other cases are intermediate. On $V_{\Gamma}$ we define the linear operator $A_{N D}$ ("Neumann-to-Dirichlet operator") by

$$
A_{N D} v:=\left.\psi\right|_{\Gamma},
$$

where $\psi$ satisfies (2.10) and $\int_{\Gamma} \psi d \Gamma=0$. Hence, again in terms of the notation (2.6), the assumption $\left.D_{v} \gamma\right|_{\Gamma}=0$ implies

$$
\left.\partial_{\varepsilon} \bar{\gamma}_{\varepsilon}\right|_{\varepsilon=0}=-\delta \nabla_{\Gamma} \gamma \nabla_{\Gamma} A_{N D} v
$$

and we obtain for

$$
\mathscr{E}^{\prime}(\gamma, \Gamma)\{v\}:=\left.\frac{d}{d \varepsilon} \mathscr{E}\left(\gamma_{\varepsilon}, \Gamma_{\varepsilon}\right)\right|_{\varepsilon=0}
$$

using again the formula for the first variation of area

$$
\mathscr{E}^{\prime}(\gamma, \Gamma)\{v\}=\int_{\Gamma}\left(\left.\partial_{\varepsilon} \bar{\gamma}_{\varepsilon}\right|_{\varepsilon=0}-\kappa \gamma v\right) d \Gamma=-\int_{\Gamma}\left(\kappa \gamma v+\delta \nabla_{\Gamma} \gamma \nabla_{\Gamma} \psi\right) d \Gamma .
$$

It easily follows from Green's formula that $A_{N D}$ is symmetric with respect to the usual $L^{2}$-scalar product on $\Gamma$, and thus

$$
\mathscr{E}^{\prime}(\gamma, \Gamma)\{v\}=-\int_{\Gamma}\left(\gamma \kappa v-\delta \Delta_{\Gamma} \gamma A_{N D} v\right) d \Gamma=-\int_{\Gamma}\left(\gamma \kappa-\delta A_{N D} \Delta_{\Gamma} \gamma\right) v d \Gamma .
$$

We have to consider (2.7), (2.11) as a differential rule for the change of surface energy in dependence on surface and energy density. They allow the computation of the energy along any path in $M$ starting from a know intial shape $\Gamma(0)$ with know energy density $\gamma_{0}$. 
But of course, in general, this computation is path-dependent, i.e. the resulting energy in the endpoint of the path will depend on the history along the whole path.

Now, as in the case of constant $\gamma$, we define the normal velocity $V_{n} \in V_{\Gamma}$ as solution of the variational problem

$$
g_{\Gamma}\left(V_{n}, v\right)=-\mathscr{E}^{\prime}(\gamma, \Gamma)\{v\} \text { for all } v \in V_{\Gamma} .
$$

Together with (2.2), (2.5), and (2.11), this yields the dynamic boundary condition

$$
\varphi+\beta \partial_{n} \varphi=\gamma \kappa-\delta A_{N D} \Delta_{\Gamma} \gamma
$$

Summarizing and using an auxiliary function $\psi$ instead of the nonlocal operator $A_{N D}$, we have obtained the following moving boundary problem: For a given bounded domain $\Omega(0) \subset \mathbb{R}^{m}$ and a given nonnegative function $\gamma_{0}$ defined on $\partial \Omega(0)$ one looks for a family of $C^{2}$-domains $\Omega(t) \subseteq \mathbb{R}^{m}, t>0$ and functions $\varphi(\cdot, t), \psi(\cdot, t) \in C^{2}(\overline{\Omega(t)}), \gamma_{t} \in C^{2}(\Gamma(t))$ such that

$$
\begin{aligned}
\Delta \varphi(\cdot, t) & =0 & & \text { in } \Omega(t), \\
\Delta \psi(\cdot, t) & =0 & & \text { in } \Omega(t), \\
\partial_{n} \psi(\cdot, t) & =\Delta_{\Gamma(t)} \gamma_{t} & & \text { on } \Gamma(t), \\
\varphi(\cdot, t)+\beta \partial_{n} \varphi(\cdot, t) & =\gamma_{t} \kappa(t)-\delta \psi(\cdot, t) & & \text { on } \Gamma(t), \\
V_{n}(t) & =\partial_{n} \varphi(\cdot, t) & & \text { on } \Gamma(t),
\end{aligned}
$$

where $\kappa(t)$ is the curvature of $\Gamma(t)$. In the main part of this paper, we restrict our attention to the case $\delta=1$. The generalization to $\delta \in[0,1)$ is sketched at the end of Section 5. Additionally, we describe the transport of $\gamma$ by (2.7), (2.8) with $\delta=1$. Introducing Lagrangian coordinates $x=x(\xi, t), \xi \in \Gamma(0)$ corresponding to the velocity field via

$$
\partial_{t} x(\xi, t)=\nabla \varphi(x(\xi, t), t) \text { for } t \geq 0, \quad x(\xi, 0)=\xi,
$$

we obtain from $(2.2)$ that $x=x(\cdot, t)$ is a diffeomorphism from $\Gamma(0)$ onto $\Gamma(t)$, and the transport law for $\gamma_{t}$ takes the form

$$
\gamma_{t}(x(\xi, t))=\gamma_{0}(\xi), \quad \xi \in \Gamma(0), t \geq 0 .
$$

In (2.12), $\varphi(\cdot, t)$ and $\psi(\cdot, t)$ are determined up to a constant only, but this is without significance for the evolution of both $\Omega(t)$ and $\gamma_{t}$. Note that in the case $\beta=0$, by setting $\Phi=\varphi+\psi,(2.12)$ simplifies to

$$
\left.\begin{array}{rlrl}
\Delta \Phi(\cdot, t) & =0 & & \text { in } \Omega(t), \\
\Phi(\cdot, t) & =\gamma_{t} \kappa(t) & & \text { on } \Gamma(t), \\
V_{n} & =\partial_{n} \Phi(\cdot, t)-\Delta_{\Gamma(t)} \gamma_{t} & & \text { on } \Gamma(t) .
\end{array}\right\}
$$

In the sequel, however, we will restrict our attention to the case $\beta>0$. Without loss of generality, we can assume $\beta=1$. In the case $\beta>0, \delta=1$, we can show well-posedness of our moving boundary problem even if $\gamma$ is zero on parts of the boundary, provided its square root is smooth. This seems to be particular to this situation. We intend to discuss the case $\beta=0$, which leads to a third-order problem, in a forthcoming paper.

For $\gamma_{t}=\gamma=$ const and $\gamma>0, \psi$ is constant, and (2.12) is known as the so-called HeleShaw flow problem with kinetic undercooling and surface tension regularization. From a 
modelling point of view, this problem can be seen as the quasistationary version of the well-known Stefan problem. In this context, the boundary condition incorporates both the Gibbs-Thomson surface energy and a nonequilibrium effect of temperature decrease at the advancing phase boundary. A short-time existence proof for this problem and a proof that its solution is the limit for the solutions of the corresponding Stefan problems can be found in [19]. For existence results concerning a corresponding two-phase problem we refer to $[5,20]$. Both effects are known to regularize the motion of the interface, and this is also true for Hele-Shaw flow problems $[12,17,18]$. In the case $\gamma \equiv 0$, with internal sources or sinks as driving forces, existence results are given in [10] for the twodimensional case and analytic data and in [15] for arbitrary dimensions, in the framework of Sobolev spaces.

If $\gamma$ is a positive constant, the moving boundary has stable, attractive equilibria which are given by the spheres (see e.g. $[4,6]$ for the case $\beta=0$ ). In general, however, after prescribing a nonconstant function $\gamma$ on the reference domain and an inital diffeomorphism $u$, it is not a priori clear (even with $\gamma$ near a constant and the moving domain near a ball) what the long-time evolution and the corresponding equilibrium will be. Instead, determining the equlibria belonging to a $\gamma$ prescribed on the reference domain leads to a stationary free boundary problem in $\psi$ whose solvability and stability (for $\Gamma$ near a sphere and $\gamma$ near a constant) we intend to discuss elsewhere.

\section{Notation and main results}

We list some notation. $C, C_{1}, \ldots$ etc. denote generic constants; their dependences on other quantities is only indicated if not obvious from the context. Let $E \subseteq \mathbb{R}^{m}, m \geq 2$ be a bounded domain with smooth boundary $S:=\partial E$ and $\nu$ the outer unit normal on $S$. For $M=S$ or $M=E$, we make constant use of the usual $L^{2}$-based Sobolev spaces $H^{s}(S), H^{s}\left(S, \mathbb{R}^{m}\right)$ of order $s$ with values in $\mathbb{R}$ and $\mathbb{R}^{m}$, respectively. If no confusion is likely, we just write $H^{s}$. The norms of these spaces will be denoted by $\|\cdot\|_{s}^{M}$; for $M=S$ the upper index $M$ is dropped in most cases. When Fréchet derivatives of operatorvalued mappings are considered, the additional arguments describing the variations are written in accolades $(\{\})$.

\subsection{Well-posedness for the moving boundary problem}

Now, as already mentioned in the introduction, we reformulate the moving boundary problem (2.12) - (2.14) by describing $\Gamma(t)$ as an embedding $u(\cdot, t): S \rightarrow \mathbb{R}^{m}$ such that the curves $t \mapsto u(y, t)$ for fixed $y \in S$ are trajectories belonging to the velocity field and $\gamma_{t}$ is constant along these curves. This approach enables us to consider $\gamma_{t}$ as a known function during the evolution at the cost of describing the moving boundary by $m$ functions. To do so, let

$$
U:=\left\{u: S \rightarrow \mathbb{R}^{m}|u=w|_{S} \text { with } w \in \operatorname{Diff}\left(\bar{E}, \Omega_{u} \cup \Gamma_{u}\right)\right\},
$$

where

$$
\Omega_{u}:=w(E) \quad \text { and } \quad \Gamma_{u}:=\partial \Omega_{u}=u(S) .
$$


Throughout this paper, we use the abbreviation

$$
U_{s}:=U \cap H^{s}\left(S, \mathbb{R}^{m}\right) .
$$

Now, (2.12) - (2.14) is reduced to the following Cauchy problem, which will be investigated in the sequel: For given $u_{0} \in U_{s}, s$ sufficiently large, we look for $T>0$ and a mapping $[0, T] \ni t \mapsto u(t) \in U_{s}$, such that

$$
\begin{aligned}
u^{\prime}(t) & =\mathscr{F}(u(t)), \quad t \in[0, T], \\
u(0) & =u_{0} .
\end{aligned}
$$

Thereby, for $u \in U$, we have set

$$
\mathscr{F}(u):=F(u)(\mathscr{G}(u)) \quad \text { with } \quad \mathscr{G}(u):=H(u)+G(u),
$$

where, for any given function $f$ on $S$,

$$
F(u) f:=\nabla \varphi(u, f) \circ u,
$$

and $\varphi=\varphi(u, f)$ denotes the solution of the Robin boundary value problem

$$
\Delta \varphi=0 \text { in } \Omega_{u}, \quad \partial_{n} \varphi+\varphi=f \circ u^{-1} \text { on } \Gamma_{u} .
$$

Further, $H(u), G(u)$ are given by

$$
H(u):=\gamma\left(\kappa_{\Gamma_{u}} \circ u\right), \quad G(u):=-A(u)(\Delta(u) \gamma) .
$$

Here $\gamma \in C^{\infty}(S)$ is a fixed and given nonnegative function, $\kappa_{\Gamma_{u}}$ denotes the mean curvature of $\Gamma_{u}$ with sign and scaling conventions as above,

$$
\Delta(u) w:=\Delta_{\Gamma_{u}}\left(w \circ u^{-1}\right) \circ u
$$

is the pullback to $S$ of the Laplace-Beltrami operator $\Delta_{\Gamma_{u}}$ on $\Gamma_{u}$ and

$$
A(u) f:=\varphi_{N}(u, f) \circ u
$$

is the Neumann-Dirichlet operator, i.e. $\varphi_{N}=\varphi_{N}(u, f)$ solves the Neumann problem

$$
\Delta \varphi_{N}=0 \text { in } \Omega_{u}, \quad \partial_{n} \varphi_{N}=c+f \circ u^{-1} \text { on } \Gamma_{u}, \quad \int_{\Gamma_{u}} \varphi_{N} d x=0 .
$$

The constant $c=c(u, f) \in \mathbb{R}$ in (3.10) is determined by the solvability condition

$$
\int_{\Gamma_{u}}(f \circ u+c) d \Gamma_{u}=0
$$

clearly $c(u, f)=0$ for $f=\Delta(u) \gamma$. For fixed smooth $\gamma$ on $S$, the mappings $u \mapsto H(u)$ and $u \mapsto \Delta(u) \gamma$ constitute quasi-linear second order differential operators on $S$. Moreover, the solutions of the boundary value problems (3.6), (3.10) depend smoothly on the domain $\Omega_{u}$, i.e. on $u \in H^{s}, s>(m+1) / 2$ and $f \mapsto F(u) f, f \mapsto A(u) f$ represent pseudodifferential operators of order zero and minus one, respectively. In particular, $G$ is a pseudodifferential operator of lower order than $H$ and may be considered as a correction term to ensure the gradient flow structure of the evolution problem. For precise formulations of the mapping properties of $F$ and $A$ and detailed proofs see Section 4 . In the consequence, this leads to

$$
[u \mapsto \mathscr{F}(u)] \in C^{\infty}\left(U_{s}, H^{s-2}\left(S, \mathbb{R}^{m}\right)\right)
$$

for $s>(m+3) / 2$. Now we are in position to formulate our main results. 
Theorem 3.1. (Short-time existence and uniqueness.)

Fix an integer $s_{0}>(m+5) / 2$ and assume $\gamma=\rho^{2}$ with $\rho \in C^{\infty}(S)$. Let $s \geq s_{0}$ be integer and $u_{0} \in U_{s}$. Then there exists $T>0$ and an unique solution

$$
u \in C\left([0, T], U_{s}\right) \cap C^{1}\left([0, T], H^{s-2}\left(S, \mathbb{R}^{m}\right)\right)
$$

of the initial value problem (3.2), (3.3). Additionally, any given $\bar{u}_{0} \in U_{s_{0}}$ has a suitable $H^{s_{0}}$-neighbourhood $K$, such that for initial values $u_{0}$ varying in $K \cap H^{s}$, there are $T>0$ and $C$ independent of $u_{0}$ such that

$$
\|u(t)\|_{s} \leq C\left(1+\|u(0)\|_{s}\right) \text { for all } t \in[0, T] .
$$

Theorem 3.2. (Regularity and continuous dependence on initial values.) Under the assumptions of Theorem 3.1 let $u$ be any solution to (3.2) in the class (3.13) with some $T>0$. Then there holds:

(i) $u(0) \in H^{s+1}\left(S, \mathbb{R}^{m}\right)$ implies

$$
u \in C\left([0, T], U_{s+1}\right) \cap C^{1}\left([0, T], H^{s-1}\left(S, \mathbb{R}^{m}\right)\right) .
$$

(ii) Assume $u_{0}^{n} \rightarrow u_{0}$ in $H^{s}\left(S, \mathbb{R}^{m}\right)$ for $n \rightarrow \infty$. Then, for $n$ sufficiently large, there exist solutions $u_{n}$ of (3.2) in the class (3.13) with initial values $u_{n}(0)=u_{0}^{n}$ and there holds $u_{n} \rightarrow u$ in $C\left([0, T], H^{s}\left(S, \mathbb{R}^{m}\right)\right)$.

The proof of both theorems is given in Section 6 .

\subsection{An existence result for abstract evolution equations}

Here we consider (3.2), (3.3) as an abstract nonlinear Cauchy problem for an unknown function $u=u(t)$ with values in a Banach space and prove existence of a solution if the nonlinearity $\mathscr{F}$ satisfies a certain condition of semi-boundedness with respect to a family of bilinear forms. As general framework we adopt the following assumptions.

Let $X \subseteq Y \subseteq Z$ be real, separable Banach spaces with dense und continuous embeddings and $\mathscr{U} \subseteq Y$ open. For every $u \in \mathscr{U}$ let $\langle\cdot, \cdot\rangle_{u}$ : $X \times Z \rightarrow \mathbb{R}$ be a continuous and nondegenerate bilinear form, such that with fixed constants $C \geq 1, M \geq 0$ :

(H1) $\langle v, w\rangle_{u}=\langle w, v\rangle_{u}$ for all $v, w \in X$;

(H2) $\quad C^{-1}\|v\|_{Y}^{2} \leq\langle v, v\rangle_{u} \leq C\|v\|_{Y}^{2}$ for all $v \in X, u \in \mathscr{U}$;

(H3) $\langle v, v\rangle_{u} \leq\langle v, v\rangle_{w}\left(1+M\|u-w\|_{Z}\right)$ for all $v \in X, u, w \in \mathscr{U}$;

(H4) weak convergences $u_{n} \rightarrow u$ in $Y, u_{n}, u \in \mathscr{U}$, and $w_{n} \rightarrow w$ in $Z$ imply $\left\langle v, w_{n}\right\rangle_{u_{n}} \rightarrow\langle v, w\rangle_{u}$ for all $v \in X$.

Assuming $(\mathrm{H})$ to hold, by the dense embedding $X \subseteq Y$ and

$$
\left|\langle v, w\rangle_{u}\right|^{2} \leq\langle v, v\rangle_{u}\langle w, w\rangle_{u} \leq C^{2}\|v\|_{Y}^{2}\|w\|_{Y}^{2} \text { for } v, w \in X
$$

there exists to each $u \in \mathscr{U}$ a scalar product $(\cdot, \cdot)_{u}$ on $Y$, which is compatible with $\langle\cdot, \cdot\rangle_{u}$, i.e. we have holds

$$
(v, w)_{u}=\langle v, w\rangle_{u} \text { for } v \in X, w \in Y .
$$


Moreover, for $u_{n}, u \in \mathscr{U}, u_{n} \rightarrow u, w_{n} \rightarrow w$ in $Y$ implies

$$
\left(v, w_{n}\right)_{u_{n}} \rightarrow(v, w)_{u} \text { for all } v \in X .
$$

In further considerations, for the sake of brevity we put

$$
\|v\|_{u}=(v, v)_{u}^{1 / 2}, \quad\|\| u \|=(u, u)_{u}^{1 / 2} .
$$

Assumption (H2) implies that $\|\cdot\|_{Y}$ and $\|\cdot\|_{u}$ are equivalent, hence $Y$ has all topological properties of a Hilbert space, in particular, $Y$ is reflexive. From $u_{n}, u \in \mathscr{U}, u_{n} \rightarrow u$ in $Y$ it follows

$$
\|u\| \leq \underline{\lim _{n \rightarrow \infty}}\left\|u_{n}\right\| \mid
$$

if $\|u\|\left\|=\lim _{n \rightarrow \infty}\left|\left\|u_{n}\right\|\right|\right.$ one concludes hereby $u_{n} \rightarrow u$ in $Y$.

Theorem 3.3. Let $(\mathrm{H})$ be valid and $\mathscr{F}: \mathscr{U} \rightarrow Z$ a weakly sequentially continuous mapping such that for every $u_{0} \in \mathscr{U}$ there exists a neighbourhood $B\left(u_{0}\right) \subset \mathscr{U}$ of $u_{0}$ in $Y$ with

$$
\sup \left\{\langle u, \mathscr{F}(u)\rangle_{u} \mid u \in B\left(u_{0}\right) \cap X\right\}<+\infty .
$$

Then for any $u_{0} \in \mathscr{U}$, there exist $T>0$ and a solution $u$ of (3.2), (3.3) in the class

$$
u \in C_{w}([0, T], \mathscr{U}) \cap C_{w}^{1}([0, T], Z) .
$$

Additionally, this solution satisfies $u(t) \rightarrow u_{0}$ in $Y$ for $t \rightarrow+0$. Moreover, $T>0$ can be chosen uniformly for initial values taken from a suitable neighbourhood of $u_{0}$ in $Y$.

In (3.16), we denote by $C_{w}([0, T], \mathscr{U})$ the space of functions from $[0, T]$ to $\mathscr{U}$ which are continuous with respect to weak convergence in $Y$. Similarly, $C_{w}^{1}([0, T], Z)$ denotes the set of weakly differentiable functions from $[0, T]$ to $Z$ with derivative in $C_{w}([0, T], Z)$. It should be noted that in general there is no uniqueness and no continuous dependence on initial data in any sense in Theorem 3.3. This theorem can be easily derived from a more elaborate, quantitative formulation given in the next theorem. Remark that, for the limit case $R=+\infty$ and bilinear forms independent of $u$, this theorem coincides with Theorem A in [13], but, as already mentioned in the introduction, our application just requires the generalization to such variable bilinear forms.

Theorem 3.4. Assume $(\mathrm{H})$ is satisfied with some ball

$$
\mathscr{U}=B:=\left\{u \in Y \mid\|u\|_{Y}<R\right\}, \quad R>0,
$$

and $\mathscr{G}: B \rightarrow Z$ is a weakly sequentially continuous mapping such that

$$
2\langle u, \mathscr{G}(u)\rangle_{u}+M\|\mathscr{G}(u)\|_{z}\|u\| \leq \beta\left(\|u\|^{2}\right) \text { for all } u \in X \cap B
$$

with a $C^{1}$-function $\beta: \mathbb{R}_{+} \rightarrow \mathbb{R}_{+}=[0, \infty)$. Let $u_{0} \in B$,

$$
|| u_{0} \mid \|<r:=R /\left(2 C^{3}\right)^{1 / 2},
$$

and $T>0$ such that the solution $\rho$ of the scalar Cauchy problem

$$
d \rho / d t=\beta(\rho(t)), \quad \rho(0)=\left\|u_{0}\right\|^{2}
$$


exists on $[0, T]$ and satisfies $\rho(t)<r^{2}$ there. Then the Cauchy problem

$$
u^{\prime}(t)=\mathscr{G}(u(t)), \quad u(0)=u_{0},
$$

possesses a solution $u$ in the class (3.16) for which additionally

$$
\begin{gathered}
\|u(t)\|^{2} \leq \rho(t) \text { for all } t \in[0, T], \\
u(t) \rightarrow u_{0} \text { in } Y \text { for } t \rightarrow+0 .
\end{gathered}
$$

The proof of this theorem will be given in the appendix.

Proof of Theorem 3.3. Let $u_{0} \in \mathscr{U}$ and $B\left(u_{0}\right)$ as in Theorem 3.3 be given. We set

$$
\begin{gathered}
\mathscr{G}(v)=\mathscr{F}\left(v+w_{0}\right) \text { for } v \in B:=\left\{v \in Y \mid\|v\|_{Y}<R\right\}, \\
\langle\cdot, \cdot\rangle_{v, 1}:=\langle\cdot, \cdot\rangle_{v+w_{0}}, \quad\|v\|_{1}:=(v, v)_{v+w_{0}}^{1 / 2},
\end{gathered}
$$

whereby the density of $X$ in $Y$ enables us to choose $w_{0} \in X$ and $R>0$ such that

$$
\left\|w_{0}-u_{0}\right\|_{Y}<R /\left(32 C^{5}\right)^{1 / 2}, \quad\left\{w_{0}+v \mid v \in B\right\} \subseteq B\left(u_{0}\right) .
$$

Clearly, the bilinear form $\langle\cdot, \cdot\rangle_{v, 1}, v \in B$ satisfies the assumptions (H) again (with same constants as $\left.\langle\cdot, \cdot\rangle_{u}, u \in \mathscr{U}\right)$. Further, by (3.15), there exists $L>0$ such that

$$
\left\langle v+w_{0}, \mathscr{G}(v)\right\rangle_{v, 1} \leq L \text { for all } v \in B \cap X
$$

and, by the weak sequential continuity of $\mathscr{F}$, the reflexivity of $Y$ and $(\mathrm{H} 4)$,

$$
\left|\left\langle w_{0}, \mathscr{G}(v)\right\rangle_{v, 1}\right|,\|\mathscr{G}(v)\|_{Z} \leq L \text { for all } v \in B .
$$

Thus

$$
2\langle v, \mathscr{G}(v)\rangle_{v, 1}+M\|\mathscr{G}(v)\|_{Z}\|v\|_{1} \leq K \text { for all } v \in B \cap X
$$

with $K:=L(4+M C R)$. Now, for any given $w \in Y$ with

$$
\left\|w-u_{0}\right\|_{Y} \leq R /\left(32 C^{5}\right)^{1 / 2},
$$

we apply Theorem 3.4 to solve the initial value problem

$$
d v / d t=\mathscr{G}(v), \quad v(0)=w-w_{0}
$$

which corresponds to (3.2) with initial value $u(0)=w$. As

$$
\left\|w-w_{0}\right\|_{1} \leq C\left(\left\|w-u_{0}\right\|_{Y}+\left\|u_{0}-w_{0}\right\|_{Y}\right)<r / 2, \quad r:=R /\left(2 C^{3}\right)^{1 / 2},
$$

Theorem 3.4 ensures the existence of a solution in the class (3.16) with $T=3 r^{2} /(4 K)$ and $v(t) \rightarrow w-w_{0}$ in $Y$ for $t \rightarrow+0$.

\section{Smooth domain dependence of the nonlocal operators}

In this Section we study the domain dependence, i.e. dependence on $u \in U_{s}$, of the Robin problem (3.6) by transforming it into a boundary value problem on the fixed reference domain $E$. In particular, we derive multilinear estimates for the Fréchet derivatives w.r. to $u$. The crucial tools here will be estimates for (multiple) pointwise products in our scale of Sobolev spaces and a differentiation rule based on invariance properties of the nonlocal operators. 
To begin with, we recall some well-known basic properties and estimates concerning Sobolev spaces. For $s>d / 2$, where $M$ is $E$ or $S$ and $d=\operatorname{dim} M / 2$, the spaces $H^{s}(M)$ turn into Banach algebras and the pointwise product of functions $w_{1}, \ldots, w_{n} \in H^{s}(M)$ allows the estimate

$$
\left\|w_{1} w_{2} \cdots w_{n}\right\|_{s}^{M} \leq C \sum_{i=1}^{n}\left(\left\|w_{i}\right\|_{s}^{M} \prod_{j \neq i}\left\|w_{j}\right\|_{s_{0}}^{M}\right)
$$

if $s \geq s_{0}>d / 2$. Moreover, for such values of $s$ the composition of $C^{\infty}$-functions with $H^{s}$-functions leads to $H^{s}$-functions again: e.g. $\Psi \in C^{\infty}(\bar{M} \times \mathbb{R})$ and $w \in H^{s}(M)$ imply $\Psi(\cdot, w(\cdot)) \in H^{s}(M)$ (note the continuity of $w$ by Sobolev's embedding),

$$
[w \mapsto \Psi(\cdot, w(\cdot))] \in C^{\infty}\left(H^{s}(M), H^{s}(M)\right)
$$

and there holds

$$
\|\Psi(\cdot, w(\cdot))\|_{s}^{M} \leq C\left(1+\|w\|_{s}^{M}\right)
$$

for all $w$ from bounded subsets of $H^{s_{0}}(M)$, where the constant depends, in addition to $s_{0}, s$ and $M$, on $\Psi$ and on upper bounds of $\|w\|_{s_{0}}$. In particular,

$$
\|1 / w\|_{s}^{M} \leq C\left(\alpha,\|w\|_{s_{0}}^{M}\right)\|w\|_{s}^{M}
$$

for all $w \in H^{s}(M)$ with $w \geq \alpha>0$ on $M$. On the other hand, we have the following counterpart of (4.1) for the product of functions $w_{1} \in H^{s_{1}}(M), \ldots, w_{n} \in H^{s_{n}}(M)$

$$
\left\|w_{1} w_{2} \cdots w_{n}\right\|_{s}^{M} \leq c\left\|w_{1}\right\|_{s_{1}}^{M}\left\|w_{2}\right\|_{s_{2}}^{M} \cdots\left\|w_{n}\right\|_{s_{n}}^{M}
$$

if $0 \leq s \leq s_{1}, \ldots, s_{n} \leq s_{0}$ with $s_{1}+\ldots+s_{n} \geq s+(n-1) s_{0}$ and $s_{0}>d / 2$.

In the following, for functions $w$ defined on $S$ let $\mathscr{E} w$ be an extension into $\bar{E}$, i.e. $\left.\mathscr{E} w\right|_{S}=w$, whereby the trace mapping theorem permits us to choose

$$
\mathscr{E} \in \mathscr{L}\left(H^{s}(S), H^{s+1 / 2}(E)\right) \text { for all } s>0 .
$$

For $\mathbb{R}^{m}$-valued functions we apply $\mathscr{E}$ componentwise.

Lemma 4.1. Let $v \in U_{s}, s>(m+1) / 2$. Then there exist a $H^{s}$-neighbourhood $V_{s} \subseteq U_{s}$ of $v$ and a map $u_{0} \in C^{\infty}\left(\bar{E}, \mathbb{R}^{m}\right)$ such that for every $u \in V_{s}$ the mapping

$$
\widetilde{u}:=u_{0}+\mathscr{E}\left(u-u_{0}\right)
$$

defines a diffeomorphism of $\bar{E}$ onto $\bar{\Omega}_{u}$.

Proof. By the definition (3.1) of $U$, every $v \in U$ has a an extension $v_{1} \in \operatorname{Diff}\left(\bar{E}, \bar{\Omega}_{v}\right)$ and there exists a $\varepsilon>0$ such that $w \in \operatorname{Diff}\left(\bar{E}, \bar{\Omega}_{\left.w\right|_{S}}\right)$ for all $w \in C^{1}\left(\bar{E}, \mathbb{R}^{n}\right)$ with $\left\|w-v_{1}\right\|_{C^{1}}^{\bar{E}} \leq \varepsilon$. Thus it suffices to find $u_{0}$ and $V_{s}$ with

$$
\left\|\widetilde{u}-v_{1}\right\|_{C^{1}}^{\bar{E}} \leq \varepsilon \text { for all } u \in V_{s},
$$

where $\widetilde{u}$ is given by (4.7). Let

$$
\mathscr{E}_{1} \in \mathscr{L}\left(C^{1}\left(S, \mathbb{R}^{m}\right), C^{1}\left(\bar{E}, \mathbb{R}^{m}\right)\right)
$$

be an extension operator which maps $C^{\infty}\left(S, \mathbb{R}^{m}\right)$ into $C^{\infty}\left(\bar{E}, \mathbb{R}^{m}\right)$. Setting

$$
u_{0}=w_{1}+\mathscr{E}_{1} w_{2} \quad \text { with } \quad w_{1} \in C^{\infty}\left(\bar{E}, \mathbb{R}^{m}\right), w_{2} \in C^{\infty}\left(S, \mathbb{R}^{m}\right)
$$


to be chosen later, we get by Sobolev embedding $H^{s+1 / 2}(E) \hookrightarrow C^{1}(\bar{E})$ and (4.6), (4.9)

$$
\begin{aligned}
\left\|\widetilde{u}-v_{1}\right\|_{C^{1}}^{\bar{E}} & \leq C\left\|u-u_{0}\right\|_{H^{s}}^{S}+\left\|u_{0}-v_{1}\right\|_{C^{1}}^{\bar{E}} \\
& \leq C\left(\left\|w_{2}\right\|_{C^{1}}^{S}+\left\|u-u_{0}\right\|_{H^{s}}^{S}\right)+\left\|w_{1}-v_{1}\right\|_{C^{1}}^{\bar{E}} \\
& \leq C\left(\left\|w_{1}-v_{1}\right\|_{C^{1}}^{\bar{E}}+\left\|w_{2}+w_{1}-v\right\|_{H^{s}}^{S}+\left\|v-u_{0}\right\|_{H^{s}}^{S}+\|u-v\|_{H^{s}}^{S}\right) .
\end{aligned}
$$

Hence, letting $\delta=\varepsilon /(4 C)$ and choosing firstly $w_{1}$ with $\left\|w_{1}-v_{1}\right\|_{C^{1}}^{\bar{E}} \leq \delta$ and, afterwards, $w_{2}$ with $\left\|w_{2}+w_{1}-v\right\|_{H^{s}}^{S} \leq \delta$ then (4.8) is valid with $V_{s}=\left\{u \mid\|u-v\|_{H^{s}}^{S}<\delta\right\}$.

Fix $s>(m+1) / 2, v \in U_{s}$, and $V_{s}$ according to Lemma 4.1. Maintaining notation and construction of this lemma, let

$$
\bar{E} \ni x \rightarrow y=\widetilde{u}(x)=\left(\widetilde{u}_{1}(x), \ldots, \widetilde{u}_{m}(x)\right) \in \bar{\Omega}_{u}, \quad u \in V_{s},
$$

be the corresponding diffeomorphism (4.7), $J=\left(\partial_{i} \widetilde{u}_{j}\right)$ its Jacobian and $\left(g_{i j}\right)=J^{\top} J$ the Euclidean metric tensor relative to the above coordinates. Furthermore let $\left(g^{i j}\right)$ be the inverse of $\left(g_{i j}\right)$ and $g=\operatorname{det}\left(g_{i j}\right)$. Then we have $\left(g^{i j}\right)=g^{-1}(\operatorname{Cof} J)^{\top}(\operatorname{Cof} J)$ where Cof $J=\left(a_{i j}\right)$ and $a_{i j}$ is the algebraic complement of $\partial_{i} \widetilde{u}_{j}$ in $J$. Note that, uniformly in $u \in V_{s}$, the function $g$ is strictly positive in E. Moreover, for the transformation $\omega=d \Gamma_{u} / d S$ of surface area elements via (4.10) and the outer normals $n$ of $\Omega_{u}$ and $\nu$ of $S$ there holds

$$
\omega=|(\operatorname{Cof} J) \nu|, \quad n \circ \widetilde{u}=(\operatorname{Cof} J) \nu /|(\operatorname{Cof} J) \nu| .
$$

By definition, all of the quantities $g, g_{i j}, a_{i j}$ and $g^{i j}$ are polynomials in the first derivatives of $\widetilde{u}$ and, in case of $g^{i j}$, additionally in $1 / g$. Consequently, remembering (4.1)-(4.5) and the construction (4.7) of $\widetilde{u}$ we obtain smooth dependence of these quantities on $u$. More precisely, we have

$$
[u \mapsto q] \in C^{\infty}\left(V_{s}, H^{s-1 / 2}(E)\right), \quad q=g, g_{i j}, a_{i j} \text { or } g^{i j}
$$

and (4.5) implies an estimate of the $k$-th Fréchet derivative:

$$
\left\|q^{(k)}(u)\left\{u_{1}, \ldots, u_{k}\right\}\right\|_{t-1 / 2}^{E} \leq C\left\|u_{1}\right\|_{s_{1}} \cdots\left\|u_{k}\right\|_{s_{k}}
$$

if $1 / 2 \leq t \leq s_{1}, \ldots, s_{k} \leq s$ and $s_{1}+\ldots+s_{k} \geq t+(k-1) s$. The constant is independent of $u \in V_{s}$ and of $u_{1} \in H^{s_{1}}(S), \ldots, u_{k} \in H^{s_{k}}(S)$. Similar arguments leads to

$$
[u \mapsto p] \in C^{\infty}\left(V_{s}, H^{s-1}(S)\right), \quad p=\omega \text { or } n \circ \widetilde{u}
$$

with an estimate of the derivatives corresponding to (4.12).

Now, introducing the transformed velocity potential $\psi=\psi(u) f=\varphi(u, f) \circ \widetilde{u}$ and the transformed Laplace and boundary operator according

$$
L(u) \psi=\partial_{i}\left(\sqrt{g} g^{i j} \partial_{j} \psi\right), \quad B(u) \psi=\omega \psi+\nu_{i} \sqrt{g} g^{i j} \partial_{j} \psi,
$$

the Robin problem (3.6) may be written as

$$
L(u) \psi=0 \text { in } E, \quad B(u) \psi=\omega f \text { on } S .
$$

Note that the values of $\psi(u) f$ in $E$ depend not only on $u$ and $f$, but also on the diffeomorphism $\tilde{u}$, i.e. on the chosen $V_{s}$. On the other hand, $\left.\psi(u) f\right|_{S}$ is completely determined 
by $u$ and $f$. The symmetry of the operator $h \mapsto \varphi(u) h$ with respect to the $L^{2}$-scalar product on $\Gamma_{u}$ implies

$$
\int_{S} w \psi(u) f d S=\int_{S} \omega f \psi(u)\left(w \omega^{-1}\right) d S
$$

(recall $\omega=d \Gamma_{u} / d S$ ) and the operator $F$ from (3.5) gets the form

$$
F(u) f=\left(F_{1}(u) f, \ldots, F_{m}(u) f\right), \quad F_{i}(u) f=a_{i j} \partial_{j} \psi(u) f / \sqrt{g} .
$$

We start the investigation of $F$ by discussing a generalized version of (4.14). Note that we have to deal with two technical difficulties here, concerning nonsmooth coefficients and uniformity of the estimates for "large" subsets of $U_{s}$. Therefore, we need the following preparation:

Lemma 4.2. Let $\Omega \subset \mathbb{R}^{m}$ be a bounded smooth domain, $x_{0} \in \Omega \chi \in C_{0}^{\infty}\left(\mathbb{R}^{m}\right)$ and

$$
\chi_{\varepsilon}(x):=\chi\left(\left(x-x_{0}\right) / \varepsilon\right), \quad x \in \mathbb{R}^{m} .
$$

Moreover, let $\mu \in H^{s}(\Omega)$ with $s>m / 2$ and $\mu\left(x_{0}\right)=0$. Then there is an $s_{1} \in(m / 2, s)$ such that

$$
\lim _{\varepsilon \downarrow 0}\left\|\chi_{\varepsilon} \mu\right\|_{s_{1}}^{\Omega}=0
$$

Proof. Note at first that

$$
\left\|\chi_{\varepsilon}\right\|_{s}^{\mathbb{R}^{m}} \leq C \varepsilon^{m / 2-s}
$$

This is immediately clear for integer $s$, the general case follows by interpolation. By Sobolev's embedding, we have $\mu \in C^{\alpha}(\bar{\Omega})$ for some $\alpha>0$, and consequently, due to $\mu\left(x_{0}\right)=0$,

$$
|\mu(x)| \leq C \varepsilon^{\alpha}, \quad x \in \operatorname{supp} \chi_{\varepsilon} \cap \Omega .
$$

Thus,

$$
\left\|\chi_{\varepsilon} \mu\right\|_{0}^{\Omega} \leq C \varepsilon^{\alpha}\left\|\chi_{\varepsilon}\right\|_{0}^{\Omega} \leq C \varepsilon^{\alpha+m / 2}
$$

and

$$
\left\|\chi_{\varepsilon} \mu\right\|_{s}^{\Omega} \leq C\left\|\chi_{\varepsilon}\right\|_{s}^{\Omega}\|\mu\|_{s}^{\Omega} \leq C \varepsilon^{m / 2-s} .
$$

The assertion follows now from interpolation.

Lemma 4.3. Let $s>(m+1) / 2, s_{0} \in((m+1) / 2, s)$ be given.

For any $v \in U_{s}$ there is an $H^{s_{0}}$-neighbourhood $V_{s_{0}}$ such that the BVP

$$
L(u) w=\partial_{i} h_{i} \text { in } E, \quad B(u) w=\omega e+\nu_{i} h_{i} \text { on } S
$$

is uniquely solvable for $u \in V_{s_{0}} \cap H^{s}\left(S, \mathbb{R}^{m}\right)$, $e \in H^{s-1}(S), h \in H^{s-1 / 2}\left(E, \mathbb{R}^{m}\right)$. Moreover, we have

$$
\|w\|_{t}^{E} \leq C\left(\|h\|_{t-1}^{E}+\|e\|_{t-3 / 2}^{S}\right)
$$

for $t \in[1, s+1 / 2]$ with $C$ independent of $h$, e , and $u \in V_{s_{0}} \cap H^{s}\left(S, \mathbb{R}^{m}\right)$.

Proof. 1. Fix $v \in U_{s}$ and choose $V_{s_{0}}$ according to Lemma 4.1. Fix $u \in V_{s_{0}} \cap H^{s}\left(S, \mathbb{R}^{m}\right)$. For $t=1$, the assertions easily seen from the variational formulation. For $t=s+1 / 2$, the assertions follow from the $H^{s}$-regularity theory of elliptic boundary value problems (with 
operators in divergence form). Our coefficients $\sqrt{g} g^{i j}$, however, are only in $H^{s-1 / 2}(E)$ which is slightly nonstandard. To prove the necessary regularity result in this case, we can proceed as in the proof of Theorem A.14 in [11], replacing the Hölder norms there by Sobolev norms. To control the error terms occurring from the freezing of coefficients, we use the estimate

$$
\left\|\mu_{i j} \partial_{j} w\right\|_{s-1 / 2}^{E} \leq C\left(\left\|\mu_{i j}\right\|_{s_{1}}^{E}\|w\|_{s+1 / 2}^{E}+\left\|\mu_{i j}\right\|_{s-1 / 2}^{E}\|w\|_{s_{1}+1 / 2}^{E}\right)
$$

(and a corresponding one for the boundary term) with $s_{1}$ from Lemma 4.2. Recalling that $\mu_{i j}$ has a form to which that lemma applies, (4.17) can be established for $t=s+1 / 2$ by a usual perturbation argument, with a constant $C=C(u)$. The general case follows by interpolation.

2. To show uniformity with respect to $u \in V_{s_{0}} \cap H^{s}\left(S, \mathbb{R}^{m}\right)$, we proceed in a similar way: For $t=s+1 / 2$, pick $u_{1}, u_{2} \in V_{s_{0}} \cap H^{s}\left(S, \mathbb{R}^{m}\right)$, denote the corresponding coefficients by $\sqrt{g_{k}} g_{k}^{i j}, k=1,2$, and estimate

$$
\begin{aligned}
\|\left(\sqrt{g_{1}} g_{1}^{i j}\right. & \left.-\sqrt{g_{2}} g_{2}^{i j}\right) \partial_{j} w \|_{s-1 / 2}^{E} \\
& \leq C\left(\left\|\widetilde{u}_{1}-\widetilde{u}_{2}\right\|_{s_{0}+1 / 2}^{E}\|w\|_{s+1 / 2}^{E}+\left\|\widetilde{u}_{1}-\widetilde{u}_{2}\right\|_{s+1 / 2}^{E}\|w\|_{s_{0}+1 / 2}^{E}\right) \\
& \leq C\left(\left\|u_{1}-u_{2}\right\|_{s_{0}}^{S}\|w\|_{s}^{E}+\|w\|_{1}^{E}\right),
\end{aligned}
$$

where an interpolation inequality has been used. A similar estimate can be given for the boundary term. After shrinking $V_{s_{0}}$ if necessary, one can show the uniformity by another perturbation argument.

Under the assumptions of Lemma 4.3, as a first trivial consequence we obtain the estimate

$$
\|\psi(u) f\|_{t}^{E},\|\psi(u) f\|_{t-1 / 2}^{S} \leq C\|f\|_{t-3 / 2}
$$

for $t \in[1, s+1 / 2]$. Note for later reference that these estimates continue to hold for $t \in[0, s+1 / 2]$, provided $s>\max \{m+1,5\} / 2$. To see this, it is sufficient to show (4.18) for $t=0$; the general case follows by interpolation again. Fix $u$, pick $\phi \in L^{2}(S)$ arbitrary and define $w \in H^{3 / 2}(E)$ by

$$
L(u) w=0 \text { in } E, \quad B(u) w=\phi \text { on } S .
$$

Then, by Green's formula rewritten in the form

$$
\int_{E}\left(\phi_{1} L(u) \phi_{2} d x-\phi_{2} L(u) \phi_{1}\right) d x=\int_{S}\left(\phi_{1} B(u) \phi_{2}-\phi_{2} B(u) \phi_{1}\right) d S
$$

and (4.17) with $t=2$,

$$
\begin{aligned}
\int_{S} \phi \psi(u) f d S & =\int_{S} B(u) w \psi(u) f d S=\int_{S} w \omega f d S \\
& \leq C\|w\|_{3 / 2}\|f\|_{-3 / 2} \leq C\|\phi\|_{1 / 2}\|f\|_{-3 / 2} .
\end{aligned}
$$

This proves the second estimate in (4.18). Analogously, picking $\zeta \in L^{2}(E)$ and defining $v \in H^{2}(E)$ by

$$
L(u) v=\zeta \text { in } E, \quad B(u) w=0 \text { on } S,
$$


we get

$$
\int_{e} \zeta \psi(u) f d x=\int_{E} L(u) w \psi(u) f d x=-\int_{S} v \omega_{f} d S \leq C\|\zeta\|_{0}\|f\|_{-3 / 2} .
$$

This proves the first estimate in (4.18).

Furthermore, concerning the smooth dependence of $\psi(u) f$ on $u$, Lemma 4.3 together with (4.11), (4.13) implies via a perturbation argument

$$
[u \mapsto \psi(u)] \in C^{\infty}\left(V_{s}, \mathscr{L}\left(H^{t-3 / 2}(S), H^{t}(E)\right)\right) .
$$

Replacing $t$ by $t-3 / 2$ and considering (4.16), this leads to

Corollary 4.4. Let $s>(m+1) / 2$ and $-1 / 2 \leq t \leq s-1$, then

$$
[u \mapsto F(u)] \in C^{\infty}\left(U_{s}, \mathscr{L}\left(H^{t}(S), H^{t}\left(S, \mathbb{R}^{m}\right)\right)\right) .
$$

Lemma 4.5. Let $s>(m+1) / 2, u \in U_{s}$ and $t \in[1, s]$ be given. Then for any choice of $s_{1}, \ldots s_{k+1} \in[t, s]$ with $s_{1}+\ldots+s_{k+1} \geq t+k s$ there exists a constant $C>0$ such that for all $f \in H^{s-1}(S)$ and all $u_{1}, \ldots, u_{k} \in H^{s}\left(S, \mathbb{R}^{m}\right)$ there holds

$$
\left\|F^{(k)}(u)\left\{u_{1}, \ldots, u_{k}\right\} f\right\|_{t-1} \leq C\left\|u_{1}\right\|_{s_{1}} \ldots\left\|u_{k}\right\|_{s_{k}}\|f\|_{s_{k+1}-1} .
$$

The constant may be chosen independently of $u$ as long as $u$ varies in $H^{s}$-bounded and closed subsets of $U_{s}$.

Proof. 1. Fix $s_{0} \in((m+1) / 2, s), v \in U_{s}$ and a neighbourhood $V_{s_{0}}$ according to Lemma 4.3. We show (4.20) with $\mathrm{C}$ independent of $u \in V_{s_{0}} \cap H^{s}\left(S, \mathbb{R}^{m}\right)$. To begin with, recall the estimate (4.18) in the form

$$
\|\nabla \psi(u) f\|_{t-1}^{S},\|\psi(u) f\|_{t+1 / 2}^{E} \leq C\|f\|_{t-1}
$$

for $t \in[1, s]$; concerning the estimate of $\nabla \psi$ along $S$ in the limit case $t=1$ note that the boundary condition allows a representation of $\nabla \psi$ as a suitable linear combination of $\psi, f$ and tangential derivatives of $\psi$. In view of (4.16) this implies the asserted estimate (4.20) for the simplest case $k=0$. To obtain similar estimates for the Fréchet derivatives $\psi^{(k)}=$ $\psi^{(k)}(u)\left\{u_{1}, \ldots, u_{k}\right\} f, k=1,2, \ldots$ we have to examine the corresponding derivatives of the coefficients in the transformed Laplacian and the boundary terms. For $\psi^{(k)}$ we get

$$
\begin{aligned}
L(u) \psi^{(k)}= & -\sum L^{(l)}(u)\left\{u_{i_{1}}, \ldots, u_{i_{j}}\right\} \psi^{(k-l)}(u)\left\{u_{i_{j+1}}, \ldots, u_{i_{k}}\right\} f \text { in } E, \\
B(u) \psi^{(k)}= & -\sum B^{(l)}(u)\left\{u_{i_{1}}, \ldots, u_{i_{j}}\right\} \psi^{(k-l)}(u)\left\{u_{i_{j+1}}, \ldots, u_{i_{k}}\right\} f \\
& +\omega^{(k)}\left\{u_{1}, \ldots, u_{k}\right\} f \text { on } S,
\end{aligned}
$$

where

$$
\begin{aligned}
& L^{(l)}(u)\left\{u_{i_{1}}, \ldots, u_{i_{j}}\right\} \varphi=\partial_{i}\left(\left(\sqrt{g} g^{i j}\right)^{(k)}\left\{u_{i_{1}}, \ldots, u_{i_{j}}\right\} \partial_{j} \varphi\right), \\
& B^{(l)}(u)\left\{u_{i_{1}}, \ldots, u_{i_{j}}\right\} \varphi=\nu_{i}\left(\sqrt{g} g^{i j}\right)^{(k)}\left\{u_{i_{1}}, \ldots, u_{i_{j}}\right\} \partial_{j} \varphi+\omega^{(l)}\left\{u_{i_{1}}, \ldots, u_{i_{j}}\right\} \varphi,
\end{aligned}
$$

and the sums are extended over $1 \leq l \leq k$ and all decompositions $i_{1}<\ldots<i_{l}$ and $i_{j+1}<\ldots<i_{k}$ of the indices $1,2, \ldots, n$. In particular, if $k=1$ we obtain for $\psi^{\prime}=$ $\psi^{\prime}(u)\left\{u_{1}\right\} f$ the boundary value problem

$$
\begin{aligned}
& L(u) \psi^{\prime}=-\partial_{i}\left(\left(\sqrt{g} g^{i j}\right)^{\prime}\left\{u_{1}\right\} \partial_{j} \psi\right) \text { in } E, \\
& B(u) \psi^{\prime}=-\nu_{i}\left(\sqrt{g} g^{i j}\right)^{\prime}\left\{u_{1}\right\} \partial_{j} \psi+\omega^{\prime}\left\{u_{1}\right\}(f-\psi) \text { on } S .
\end{aligned}
$$


Thus, for any $t \in[1, s]$, Lemma 4.3 implies

$$
\left\|\psi^{\prime}(u)\left\{u_{1}\right\} f\right\|_{t+1 / 2}^{E} \leq C\left(\left\|\left(\sqrt{g} g^{i j}\right)^{\prime}\left\{u_{1}\right\} \partial_{j} \psi\right\|_{t-1 / 2}^{E}+\left\|\omega^{\prime}\left\{u_{1}\right\}(f-\psi)\right\|_{t-1}^{S}\right) .
$$

To estimate the terms on the right-hand side we obtain by (4.5)

$$
\left\|\left(\sqrt{g} g^{i j}\right)^{\prime}\left\{u_{1}\right\} \partial_{j} \psi\right\|_{t-1 / 2}^{E} \leq C\left\|\left(\sqrt{g} g^{i j}\right)^{\prime}\left\{u_{1}\right\}\right\|_{s_{1}-1 / 2}^{E}\left\|\partial_{j} \psi\right\|_{s_{2}-1 / 2}^{E},
$$

and accordingly

$$
\left\|\omega^{\prime}\left\{u_{1}\right\}(f-\psi)\right\|_{t-1}^{S} \leq C\left\|\omega^{\prime}\left\{u_{1}\right\}\right\|_{s_{1}-1}^{S}\left(\|f\|_{s_{2}-1}+\|\psi\|_{s_{2}-1}^{S}\right)
$$

for any choice of $s_{1}, s_{2} \in[t, s]$ with $s_{1}+s_{2} \geq t+s$. As

$$
\|\psi\|_{s_{2}-1}^{S},\left\|\partial_{j} \psi\right\|_{s_{2}-1 / 2}^{E} \leq C\|\psi\|_{s_{2}+1 / 2}^{E} \leq C^{\prime}\|f\|_{s_{2}-1},
$$

by (4.21), using (4.12), (4.13) we find

$$
\left\|\left(\sqrt{g} g^{i j}\right)^{\prime}\left\{u_{1}\right\} \partial_{j} \psi\right\|_{t-1 / 2}^{E},\left\|\omega^{\prime}\left\{u_{1}\right\}(f-\psi)\right\|_{t-1}^{S} \leq C^{\prime}\left\|u_{1}\right\|_{s_{1}}\|f\|_{s_{2}-1},
$$

hence

$$
\left\|\nabla \psi^{\prime}(u)\left\{u_{1}\right\} f\right\|_{t-1}^{S},\left\|\psi^{\prime}(u)\left\{u_{1}\right\} f\right\|_{t+1 / 2}^{E} \leq C\left\|u_{1}\right\|_{s_{1}}\|f\|_{s_{2}-1},
$$

where the same remark applies to the estimate of $\nabla \psi^{\prime}$ along $S$ as to (4.21). Using (4.22), these estimates are extended inductively to $\psi^{(k)}$ :

$$
\left\|\nabla \psi^{(k)}\right\|_{t-1}^{S},\left\|\psi^{(k)}\right\|_{t+1 / 2}^{E} \leq C\left\|u_{1}\right\|_{s_{1}} \cdots\left\|u_{k}\right\|_{s_{k}}\|f\|_{s_{k+1}-1},
$$

provided $s_{1}, \ldots, s_{k+1} \in[t, s]$ with $s_{1}+\ldots+s_{k+1} \geq t+k s$. In view of (4.16), these estimates together with (4.11) and (4.5) imply the asserted estimate (4.20).

2. Let $K \subset U_{s}$ be closed and bounded in $H^{s}\left(S, \mathbb{R}^{m}\right)$. As shown in 1 ., $K$ can be covered by $H^{s_{0}}$-open sets $V_{s_{0}, v}, v \in K$, such that (4.20) holds uniformly for $u \in V_{s_{0}, v} \cap K$. Now the assertion follows from the compactness of $K$ in $H^{s}\left(S, \mathbb{R}^{m}\right)$.

Now we use invariance properties with respect to diffeomorphisms (cf. e.g. [9]). Let $\tau \in \operatorname{Diff}(S)$. Then by definition

$$
\varphi(u, f)=\varphi(u \circ \tau, f \circ \tau) \text { in } \Omega_{u} .
$$

Recalling the definition of $F$, we have

$$
\begin{gathered}
(F(u) f) \circ \tau=(\nabla \varphi(u, f)) \circ(u \circ \tau), \\
F(u \circ \tau)(f \circ \tau)=(\nabla \varphi(u \circ \tau, f \circ \tau)) \circ(u \circ \tau),
\end{gathered}
$$

consequently (4.25) implies

$$
(F(u) f) \circ \tau=F(u \circ \tau)(f \circ \tau) \text { on } S .
$$

Any smooth vector field $D$ on $S$, identified with a first order differential operator, generates a one-parameter group of smooth diffeomorphisms $t \mapsto \tau_{t}$ with $\tau_{t}=i d$ for $t=0$. Setting $\tau=\tau_{t}$ in (4.26) and differentiating with respect to $t$ at $t=0$ gives

$$
D F(u) f=F^{\prime}(u)\{D u\} f+F(u) D f
$$

for $u \in U_{s}$ and $f \in H^{s-1}(S), s>(m+1) / 2$. Furthermore, by differentiation with respect 
to $u$,

$$
\begin{aligned}
D F^{(k)}(u)\{\ldots\} f= & F^{(k+1)}(u)\{D u, \ldots\} f+F^{(k)}(u)\{\ldots\} D f \\
& +\sum_{j=1}^{k} F^{(k)}(u)\left\{u_{1}, \ldots, u_{j-1}, D u_{j}, u_{j+1}, \ldots, u_{k}\right\} f,
\end{aligned}
$$

where the dots indicate the arguments $u_{1}, \ldots, u_{k} \in H^{s}\left(S, \mathbb{R}^{m}\right)$. We choose $m$ smooth vector fields $D_{1}, \ldots, D_{m}$ on $S$ such that

$$
\operatorname{span}\left\{D_{1}, \ldots, D_{m}\right\}=T_{x} \text { for all } x \in S
$$

and use the multi-index notation $D^{\alpha}=D_{1}^{\alpha_{1}} \ldots D_{m}^{\alpha_{m}}, \alpha=\left(\alpha_{1}, \ldots, \alpha_{m}\right)$ for higher order derivatives. Note that, for $s \geq 0$ integer, we can use

$$
(u, v)_{s}=\sum_{|\alpha| \leq s}\left(D^{\alpha} u, D^{\alpha} v\right)_{L^{2}(S)}
$$

as scalar product generating the norm in $H^{s}(S)$. As a consequence of $(4.27),(4.28)$ by induction we obtain a differentiation rule which resembles Leibniz' rule at an abstract level: For any multi-index $\alpha$ and $u \in U_{s}, f \in H^{s-1}(S), s>|\alpha|+(m+1) / 2$ there holds

$$
D^{\alpha} F(u) f=\sum c_{\beta_{1}, \ldots, \beta_{k+1}} F^{(k)}(u)\left\{D^{\beta_{1}} u, \ldots, D^{\beta_{k}} u\right\} D^{\beta_{k+1}} f,
$$

where the sum has to be extended over all integers $k$ and systems of nonnegative multiindices $\beta_{1}, \ldots, \beta_{k+1}$ with

$$
0 \leq k \leq|\alpha|, \quad 1 \leq\left|\beta_{1}\right|, \ldots,\left|\beta_{k}\right|, \quad \beta_{1}+\ldots+\beta_{k+1}=\alpha .
$$

The coefficients are nonnegative integers, in particular, $c_{\alpha}=c_{\alpha, 0}=1$. Combining the differentiation rule with the estimate of the derivatives in lower norms we obtain

Proposition 4.6. (i) Let $s \geq s_{0}>(m+1) / 2$ integer, $u \in U_{s+1}$. Then

$$
\|F(u) f\|_{s} \leq C\left(\|u\|_{s+1}\|f\|_{s_{0}}+\|f\|_{s}\right) .
$$

(ii) Assume additionally $s \geq s_{0}+1$ and let $\alpha$ be any multi-index with $|\alpha|=s$. Then we have

$$
D^{\alpha} F(u) f=F(u) D^{\alpha} f+F^{\prime}(u)\left\{D^{\alpha} u\right\} f,+R_{\alpha}(u) f
$$

where the remainder term allows the estimate

$$
\left\|R_{\alpha}(u) f\right\|_{0} \leq C\left(\|u\|_{s}\|f\|_{s_{0}}+\|f\|_{s-1}\right) .
$$

The constants in both estimates can be chosen uniformly as u varies in $H^{s_{0}}$ - closed, $H^{s_{0}+1}$ - bounded subsets of $U_{s+1}$.

Proof. We consider the more complicated situation (ii) only. According to (4.29), the remainder term has a representation as a sum of terms

$$
I_{\beta}=F^{(k)}(u)\left\{D^{\beta_{1}} u, \ldots, D^{\beta_{k}} u\right\} D^{\beta_{k+1}} f,
$$

where the multi-indices satisfy (4.30) and additionally $\left|\beta_{i}\right|<s=|\alpha|$. Thus $k \geq 1$ and $\left|\beta_{i}\right| \geq 1$ for at least two indices, say $i=i_{1}, i_{2}$. We estimate $I_{\beta}$ using (4.20) with 
$s_{i}=1+\left(1-\theta_{i}\right)\left(s_{0}-1\right)$ and

$$
\theta_{i}=\left(\left|\beta_{i}\right|-1\right) /(s-2), \quad i=i_{1}, i_{2}, \quad \theta_{i}=\left|\beta_{i}\right| /(s-2), \quad i \notin\left\{i_{1}, i_{2}\right\} .
$$

Then $s_{i} \in\left[1, s_{0}\right]$ and $s_{1}+\ldots+s_{k+1}=1+k s_{0}$, hence applying (4.20) (with $t=1, s=s_{0}$ ) yields

$$
\left\|I_{\beta}\right\|_{0} \leq C\|u\|_{\left|\beta_{1}\right|+s_{1}} \ldots\|u\|_{\left|\beta_{1}\right|+s_{k}}\|f\|_{\left|\beta_{k+1}\right|+s_{k+1}-1}
$$

Note that $\theta_{1}+\ldots+\theta_{k+1}=1$ and set $\lambda:=\theta_{1}+\ldots+\theta_{k}$. From

$$
\left|\beta_{i}\right|+s_{i} \leq\left(1-\theta_{i}\right)\left(s_{0}+1\right)+\theta_{i}(s-1)
$$

we get by norm convexity and Youngs inequality

$$
\begin{aligned}
\left\|I_{\beta}\right\|_{0} & \leq C\|u\|_{s_{0}+1}^{k-1}\left(\|u\|_{s_{0}+1}\|f\|_{s-1}\right)^{1-\lambda}\left(\|u\|_{s}\|f\|_{s_{0}}\right)^{\lambda} \\
& \leq C\|u\|_{s_{0}+1}^{k-1}\left(\|u\|_{s_{0}+1}\|f\|_{s-1}+\|u\|_{s}\|f\|_{s_{0}}\right) .
\end{aligned}
$$

This proves the assertion.

We conclude this section with remarks concerning the Neumann-Dirichlet operator $A$ defined by (3.9), (3.10). It is obvious that the regularity properties of $u \mapsto A(u)$ are the same as for $\left.u \mapsto \psi(u)\right|_{S}$, hence (4.19) reappears as

$$
[u \mapsto A(u)] \in C^{\infty}\left(U_{s}, \mathscr{L}\left(H^{t}(S), H^{t+1}(S)\right)\right)
$$

for $s>(m+1) / 2$ and $-1 / 2 \leq t \leq s-1$. Moreover, the differentiation rule (4.28) also holds for $A$ and $\psi_{N}(u) f:=\varphi_{N}(u) f \circ \tilde{u}$ satisfies estimates parallel to (4.24), hence we get

$$
\left\|A^{(k)}(u)\left\{u_{1}, \ldots, u_{k}\right\} f\right\|_{t} \leq C\left\|u_{1}\right\|_{s_{1}} \cdots\left\|u_{k}\right\|_{s_{k}}\|f\|_{s_{k+1}-1},
$$

provided $s_{1}, \ldots, s_{k+1} \in[t, s]$ with $s_{1}+\ldots+s_{k+1} \geq t+k s$. Thus we have the following analogon of Proposition 4.6:

Proposition 4.7. (i) Let $s \geq s_{0}>(m+1) / 2$ integer, $u \in U_{s}$ and $f \in H^{s-1}(S)$. Then

$$
\|A(u) f\|_{s} \leq C\left(\|u\|_{s}\|f\|_{s_{0}-1}+\|f\|_{s-1}\right) .
$$

with a uniform constant as long as $u$ varies in $H^{s_{0}}$-closed, $H^{s_{0}}$-bounded subsets of $U_{s}$.

(ii) Assume additionally $s \geq s_{0}+1$ and let $\alpha$ be any multi-index with $|\alpha|=s-1$. Then we have

$$
\left\|D^{\alpha} A(u) f-A(u) D^{\alpha} f\right\|_{1} \leq C\left(\|u\|_{s}\|f\|_{s_{0}-1}+\|f\|_{s-2}\right) .
$$

where now the constant can be chosen uniformly as u varies in $H^{s_{0}}$-closed, $H^{s_{0}+1}$-bounded subsets of $U_{s+1}$.

Finally, for later reference we point out the simple commutator estimate

$$
\|A(u) h f-h A(u) f\|_{1} \leq C\|h\|_{s}\|f\|_{-1}
$$

for $u \in U_{s}, f, h \in H^{s}(S), s>(m+1) / 2$. Fixing any neighbourhood $V_{s}$ according to Lemma 4.1, $u \in V_{s}$ with corresponding diffeomorphism (4.10) and considering

$$
A(u) f=\left.\psi(u)(f-\omega A(u) f)\right|_{S},
$$

(4.33) is reduced to

$$
\|h \psi(u) f-\psi(u)(h f)\|_{1}^{S} \leq C\|h\|_{s}\|f\|_{-1} .
$$


Let $\tilde{h}$ be the extension of $h$ into $E$ determined by solving the Dirichlet problem

$$
L(u) \tilde{h}=0 \text { in } E, \quad \tilde{h}=h \text { on } S .
$$

Clearly $\|\tilde{h}\|_{s+1 / 2}^{E} \leq C\|h\|_{s}$ and $\tilde{\psi}:=h \psi(u) f-\psi(u)(h f)$ solves the BVP

$$
\begin{aligned}
& L(u) \tilde{\psi}=2 \partial_{i}\left(\sqrt{g} g^{i j} \partial_{i} \tilde{h} \psi(u) f\right) \text { in } E, \\
& B(u) \tilde{\psi}=-\omega \nu_{i} g^{i j} \partial_{i} \tilde{h} \psi(u) f \text { on } S .
\end{aligned}
$$

Hence by Lemma 4.3

$$
\|\tilde{\psi}\|_{3 / 2}^{E} \leq C\left\|\sqrt{g} g^{i j} \partial_{i} \tilde{h}\right\|_{s-1 / 2}\|\psi(u) f\|_{1 / 2}^{E} \leq C\|h\|_{s}\|\psi(u) f\|_{1 / 2}^{E} .
$$

Together with (4.18) this implies (4.34).

\section{The main estimate}

In this section we prove $H^{s}$ - a priori estimates for the nonlinear operator $\mathscr{F}$ w.r. to variable bilinear forms which we define in the sequel. As already mentioned in the introduction, these estimates are the main ingredient in the existence proof.

To begin with, for $u \in U_{s}, s>(m+1) / 2$ we define

$$
\begin{gathered}
P(u) v:=v \cdot(n(u) \circ u), \quad N(u) w:=w(n(u) \circ u), \\
\Lambda(u) w:=\nabla_{\Gamma_{u}}\left(w \circ u^{-1}\right) \circ u
\end{gathered}
$$

as the euclidean scalar product and multiplication with outer normal $n(u)$ of $\Gamma_{u}$ and pullback of tangential gradient $\nabla_{\Gamma_{u}}$ along $\Gamma_{u}$, respectively. If $P(u), N(u)$, and $\Lambda(u)$ are considered as operators in $v$ and $w$, their coefficients are smooth functions of $u$ and its first derivatives. Thus, using (4.1) - (4.5),

$$
\begin{gathered}
P(u) \in \mathscr{L}\left(H^{t}\left(S, \mathbb{R}^{m}\right), H^{t}(S)\right), \quad N(u) \in \mathscr{L}\left(H^{t}(S), H^{t}\left(S, \mathbb{R}^{m}\right)\right), \\
\Lambda(u) \in \mathscr{L}\left(H^{t}(S), H^{t-1}\left(S, \mathbb{R}^{m}\right)\right)
\end{gathered}
$$

depend smoothly on $u \in U_{s}$ for $0 \leq t \leq s$ and $1 \leq t \leq s$ respectively. Clearly, the operators $P, N, \Lambda$ satisfy invariance properties as stated for $F$ in (4.26). As a consequence, the differentiation rule (4.27) and its corollary (4.28) are also true for $P, N, \Lambda$; we make use of that without explicit mention. Further, recall that the pullback $\Delta(u) w$ of the Laplace Beltrami operator $\Delta_{\Gamma_{u}}$ on $\Gamma_{u}$ according to (3.8) and the operator $H(u)$ according to (3.7) may be expressed as

$$
\Delta(u) w=\Lambda_{i}(u)\left(\Lambda_{i}(u) w\right), \quad H(u)=-\gamma \Lambda_{i}(u)\left(n_{i}(u) \circ u\right),
$$

respectively (see e.g. [8], Sect. 15.1). Thus, recalling (4.31), the operator $\mathscr{G}$ defined by (3.4), (3.7) satisfies

$$
[u \mapsto \mathscr{G}(u)] \in C^{\infty}\left(U_{s}, H^{s-2}(S)\right)
$$

provided $s>(m+3) / 2$. Together with Corollary 4.4 this implies the smoothness of $\mathscr{F}$ as stated in (3.12).

In further considerations of this section we fix the integer $s_{0}:=[(m+5) / 2]+1$ and set

$$
\widetilde{U}_{s}:=U_{s} \cap K \text { for all } s \geq s_{0}
$$


with an $H^{s_{0}}$ - bounded and $L^{2}$-closed subset $K \subseteq U_{s_{0}}$. Note that

$$
1 \leq C\|u\|_{s_{0}} \leq C^{\prime}\|u\|_{s}, \quad\|u\|_{C^{3}(S)} \leq C
$$

for all $u \in \widetilde{U}_{s}, s \geq s_{0}$. By transforming the well-know integration by parts formula for the differential operator $\nabla_{\Gamma_{u}}$ onto $S$, it gets the form

$$
\int_{S} \omega \Lambda_{i}(u) f d S=-\int_{S} \omega\left(\kappa_{\Gamma_{u}} \circ u\right)\left(n_{i}(u) \circ u\right) f d S .
$$

Consequently, for $u \in \widetilde{U}_{s}, s \geq s_{0}$ and any $f \in C^{1}(S)$ we have

$$
\left|\int_{S} \Lambda_{i}(u) f d S\right| \leq C \int_{S}|f| d S
$$

Furthermore, note the estimates

$$
\|\mathscr{G}(u)\|_{s-2},\|\mathscr{F}(u)\|_{s-2} \leq C\|u\|_{s} \text { for all } u \in \widetilde{U}_{s}, s \geq s_{0} .
$$

Now we can formulate

Lemma 5.1. Let $s \geq s_{0}$. Then for $u \in U_{s}, v \in H^{s}\left(S, \mathbb{R}^{m}\right)$ and $f \in H^{s-1}(S)$ there holds

$$
F^{\prime}(u)\{v\} f=F(u)(\Lambda(u)(P(u) v) \cdot F(u) f)+R(u)\{v\} f,
$$

where $R$ allows the estimate

$$
\|R(u)\{v\} f\|_{0} \leq C\|f\|_{s-1}\|v\|_{0} .
$$

The constant is independent of $u$ as long as u varies in $\widetilde{U}_{s}$.

Proof. As in the proof of Lemma 4.5 we can assume $u \in V_{s}$. We have

$$
F_{i}^{\prime}(u)\{v\} f=\partial_{i} \varphi^{\prime} \circ u+v_{j} \partial_{i} \partial_{j} \varphi \circ u,
$$

where $\varphi^{\prime}=\varphi^{\prime}(u, f)\{v\}$ denotes the derivative with respect to $u$ of the velocity potential $\varphi=\varphi(u, f)$ in $\Omega_{u}$. As

$$
\|\varphi(u, f)\|_{C^{2}\left(\bar{\Omega}_{u}\right)} \leq C_{1}\|\psi(u) f\|_{C^{2}(\bar{E})} \leq C_{2}\|\psi(u) f\|_{H^{s+1 / 2}(E)} \leq C_{3}\|f\|_{s-1}
$$

by Sobolev's embedding and (4.21), we obtain

$$
\left\|v_{j} \partial_{i} \partial_{j} \varphi \circ u\right\|_{0}^{S} \leq C\|f\|_{s-1}\|v\|_{0} .
$$

Furthermore, $\varphi^{\prime}$ satisfies $\Delta \varphi^{\prime}=0$ in $\Omega_{u}$ and the boundary condition

$$
\varphi^{\prime}+n \cdot \nabla \varphi^{\prime}+n^{\prime} \cdot \nabla \varphi+\left(\partial_{i} \varphi+n_{j} \partial_{i} \partial_{j} \varphi\right) v_{i} \circ u^{-1}=0 \text { on } \Gamma_{u}
$$

where we have used the abbreviation

$$
n^{\prime}=\left.\partial_{\varepsilon}\left(n(u+\varepsilon v) \circ\left(i d+\varepsilon v \circ u^{-1}\right)\right)\right|_{\varepsilon=0}
$$

for the variation of the outer normal on $\Gamma_{u}$. A simple calculation, cf. Lemma 1.1 in [3], shows

$$
n^{\prime}=-\nabla_{\Gamma_{u}}\left(n \cdot v \circ u^{-1}\right)+v_{i} \circ u^{-1} \nabla_{\Gamma_{u}} n_{i} .
$$

By retransformation onto the reference domain $E$, this implies for $\tilde{\psi}^{\prime}=\varphi^{\prime} \circ u$ to satisfy the BVP

$$
L(u) \tilde{\psi}^{\prime}=0 \text { in } E, \quad B(u) \tilde{\psi}^{\prime}=\Lambda(u)(P(u) v) \cdot F(u) f+R_{1}(u)\{v\} f \text { on } S .
$$


The operator $R_{1}$ acts by pointwise multiplications with respect to the components of $v$, hence by the same reasoning as above we get the estimate

$$
\left\|R_{1}(u)\{v\} f\right\|_{0}^{S} \leq C\|f\|_{s-1}\|v\|_{0} .
$$

Thus the result follows.

For $u \in U_{s}$ let $M(u) \in \mathscr{L}\left(L^{2}\left(S, \mathbb{R}^{m}\right)\right)$ be the operator defined by

$$
M(u) v:=v-\Lambda(u)(\psi(u) P(u) v) .
$$

By (4.19) and (5.4),

$$
M(u) \in \mathscr{L}\left(H^{t}\left(S, \mathbb{R}^{m}\right), H^{t}\left(S, \mathbb{R}^{m}\right)\right), \quad 0 \leq t \leq s-1,
$$

depends smoothly on $u \in U_{s}, s>(m+1) / 2$; for later reference we state explicitly

$$
\left\|M(u)\left\{u_{1}, \ldots, u_{k}\right\} v\right\|_{t} \leq C\left\|u_{1}\right\|_{s} \cdots\left\|u_{k}\right\|_{s}\|v\|_{t} .
$$

Because of $P(u) \Lambda(u)=0$ the operator $M(u)$ constitutes an isomorphism in $L^{2}\left(S, \mathbb{R}^{m}\right)$ with inverse

$$
M(u)^{-1} v=v+\Lambda(u)(\psi(u) P(u) v)
$$

and we have

$$
C^{-1}\|v\|_{0} \leq\|M(u) v\|_{0} \leq C\|v\|_{0}
$$

for all $v \in L^{2}\left(S, \mathbb{R}^{m}\right)$ with a positive constant $C$ independent of $u \in \widetilde{U}_{s}$.

Lemma 5.2. Let $s>(m+3) / 2$. There exists a positive constant $C$ such that for all $u \in \widetilde{U}_{s}$ and $f \in L^{2}(S), w \in L^{2}\left(S, \mathbb{R}^{m}\right)$

$$
\left|(M(u) F(u) f, M(u) w)_{0}-(f, P(u) w)_{0}\right| \leq C\|f\|_{-1}\|w\|_{0} .
$$

Proof. Reformulating the boundary condition satisfied by $\psi(u) f$, we have

$$
P(u)(F(u) f)=f-\psi(u) f, \quad F(u) f-\Lambda(u)(\psi(u) f)=N(u)(f-\psi(u) f),
$$

and consequently

$$
\begin{aligned}
M(u) F(u) f & =F(u) f-\Lambda(u) \psi(u)(f-\psi(u) f) \\
& =N(u)(f-\psi(u) f)+\Lambda(u) \psi(u)^{2} f .
\end{aligned}
$$

Further, recalling $P(u) \Lambda(u)=0$,

$$
(N(u) f, M(u) w)_{0}=(f, P(u) M(u) w)_{0}=(f, P(u) w)_{0},
$$

and we obtain

$$
(M(u) F(u) f, M(u) w)_{0}=(f-\psi(u) f, P(u) w)_{0}+\left(\Lambda(u) \psi(u)^{2} f, M(u) w\right)_{0} .
$$

Together with

$$
\left\|\Lambda(u) \psi(u)^{2} f\right\|_{0}^{S},\|\psi(u) f\|_{0}^{S} \leq C\|f\|_{-1}
$$

from (4.18), this immediately implies (5.11). 
In view of (5.10), for every fixed $u \in U_{s}, s \geq s_{0}$,

$$
(v, w)_{s, u}:=(M(u) v, M(v) w)_{0}+\sum_{|\alpha|=s}\left(M(u) D^{\alpha} v, M(u) D^{\alpha} w\right)_{0}
$$

defines a scalar product on $H^{s}\left(S, \mathbb{R}^{m}\right)$ which is equivalent to the usual one.

Lemma 5.3. Let $s \geq s_{0}$ and $u \in \widetilde{U}_{s}$.

(i) There exists a $C>0$ independent of $u$ such that for all $v \in H^{s+2}\left(S, \mathbb{R}^{m}\right)$ and $w \in H^{s}\left(S, \mathbb{R}^{m}\right)$

$$
(v, w)_{s, u} \leq C\|v\|_{s+2}\|w\|_{s-2} .
$$

(ii) There exist $\lambda_{0}, c_{0}>0$ independent of $u$ such that for all $v \in H^{s+4}\left(S, \mathbb{R}^{m}\right)$ and $\lambda \geq \lambda_{0}$

$$
\left(v,\left(\Delta_{0}^{2}+\lambda\right) v\right)_{s, u} \geq c_{0}\|v\|_{s+2}^{2} .
$$

with the elliptic operator $\Delta_{0}:=D_{i} D_{i}$ on $S$.

Proof. (i) We consider a typical term of (5.12) and show

$$
I_{\alpha}(v, w):=\left(M(u) D^{\alpha} v, M(u) D^{\alpha} w\right)_{0} \leq C\|v\|_{s+2}\|w\|_{s-2}
$$

for smooth $v, w$ and multi-indices $\alpha$ with $|\alpha|=s$. Recalling (5.8), we have

$$
\left\|M^{(k)}(u)\left\{D^{\alpha_{1}} u, \ldots, D^{\alpha_{k}} u\right\} w\right\|_{0} \leq C\|w\|_{0}
$$

if $\left|\alpha_{1}\right|, \ldots,\left|\alpha_{k}\right| \leq 2$. Thus, writing $D^{\alpha} w=D^{\beta} D^{\delta} w$ with $|\beta|=2$ and $|\delta|=s-2$, multiple application of the differentiation rule gives a representation

$$
M(u) D^{\alpha} w=\sum(-1)^{\left|\alpha_{0}\right|} D^{\alpha_{0}} M^{(k)}(u)\left\{D^{\alpha_{1}} u, \ldots, D^{\alpha_{k}} u\right\} D^{\delta} w,
$$

where $\left|\alpha_{i}\right| \leq 2$ (in fact $\alpha_{0}+\ldots+\alpha_{k}=\beta$ ), hence

$$
\left\|M^{(k)}(u)\left\{D^{\alpha_{1}} u, \ldots, D^{\alpha_{k}} u\right\} D^{\delta} w\right\|_{0} \leq C\|w\|_{s-2} .
$$

Furthermore, using the differentiation rule again, we have

$$
\left\|M(u) D^{\alpha} v\right\|_{2} \leq C\|v\|_{s+2}
$$

and consequently

$$
\left(M(u) D^{\alpha} v, D^{\alpha_{0}} M^{(k)}(u)\left\{D^{\alpha_{1}} u, \ldots, D^{\alpha_{k}} u\right\} D^{\delta} v\right)_{0} \leq C\|v\|_{s+2}\|w\|_{s-2} .
$$

This implies (5.15).

(ii) Using the same type of arguments as in the proof of part (i) we obtain

$$
\left(v, \Delta_{0}^{2} v\right)_{s, u} \geq\left(D_{i} D_{j} v, D_{i} D_{j} v\right)_{s, u}-C\|v\|_{s+1}\|v\|_{s+2}
$$

and consequently

$$
\left(v,\left(\Delta_{0}^{2}+\lambda\right) v\right)_{s, u} \geq c_{0}\left(\|v\|_{s+2}^{2}+\lambda\|v\|_{s}^{2}\right)-C\|v\|_{s+1}^{2}
$$

with a positive constant $c_{0}$. Hence applying

$$
\|v\|_{s+1}^{2} \leq \sigma\|v\|_{s+2}^{2}+C(\sigma)\|v\|_{s}^{2}
$$

with $\sigma=c_{0} / 2$ and choosing $\lambda$ sufficiently large we get the claim. 
An immediate consequence of Lemma 5.3, (i) is the existence of a continuous bilinear form $\langle\cdot, \cdot\rangle_{s, u}$ on $H^{s+2}\left(S, \mathbb{R}^{m}\right) \times H^{s-2}\left(S, \mathbb{R}^{m}\right)$ compatible with $(\cdot, \cdot)_{s, u}$, i.e. there holds $\langle v, w\rangle_{s, u}=(v, w)_{s, u}$ for all $v, w \in H^{s+2}\left(S, \mathbb{R}^{m}\right)$. Further, we put for $\varepsilon \in(0,1]$

$$
\langle v, w\rangle_{s, u}^{\varepsilon}:=\langle v, w\rangle_{s_{0}, u}+\varepsilon^{2}\langle v, w\rangle_{s, u} .
$$

Lemma 5.4. We assume as above $s \geq s_{0}, \varepsilon \in(0,1]$.

(i) For fixed $u \in U_{s}$, the mapping $\langle\cdot, \cdot\rangle_{s, u}^{\varepsilon}: H^{s+2}\left(S, \mathbb{R}^{m}\right) \times H^{s-2}\left(S, \mathbb{R}^{m}\right) \rightarrow \mathbb{R}$ constitutes a continuous, nondegenerate bilinear form, symmetric on $H^{s+2}\left(S, \mathbb{R}^{m}\right) \times$ $H^{s+2}\left(S, \mathbb{R}^{m}\right)$.

(ii) With constants $C>0$ independent of $\varepsilon, u, v, w$, one has for $u, w \in \tilde{U}_{s}$ and $v \in$ $H^{s+2}\left(S, \mathbb{R}^{m}\right)$ :

$$
\begin{gathered}
C\left(\|v\|_{s_{0}}^{2}+\varepsilon^{2}\|v\|_{s}^{2}\right) \leq\langle v, v\rangle_{s, u}^{\varepsilon} \leq C^{-1}\left(\|v\|_{s_{0}}^{2}+\varepsilon^{2}\|v\|_{s}^{2}\right) \\
\langle v, v\rangle_{s, u}^{\varepsilon} \leq\langle v, v\rangle_{s, w}^{\varepsilon}\left(1+C\|u-w\|_{s_{0}-2}\right) .
\end{gathered}
$$

(iii) The weak convergences $u_{n} \rightarrow u \in H^{s}, w_{n} \rightarrow w \in H^{s-2}$ imply for each $v \in H^{s+2}$

$$
\left\langle v, w_{n}\right\rangle_{s, u_{n}}^{\varepsilon} \rightarrow\langle v, w\rangle_{s, u}^{\varepsilon}
$$

Proof. (i) It remains to show nondegeneracy only. First note that Lemma 5.3, (ii) implies for every $v \in H^{s+2}$ and $\lambda \geq \lambda_{0}$

$$
\left\langle v, \Delta_{0}^{2} v+\lambda v\right\rangle_{s, u}^{\varepsilon} \geq c_{0}\|v\|_{0}^{2} .
$$

Let $\varepsilon, u, w$ be fixed such that $\langle v, w\rangle_{s, u}^{\varepsilon}=0$ for every $v \in H^{s+2}$. Let $\lambda$ be sufficiently large and $v \in H^{s+2}$ the unique solution of the fourth-order elliptic equation

$$
\Delta_{0}^{2} v+\lambda v=w \text { on } S .
$$

Thus we have

$$
0=\langle v, w\rangle_{s, u}^{\varepsilon}=\left\langle v, \Delta_{0}^{2} v+\lambda v\right\rangle_{s, u}^{\varepsilon} \geq c_{0}\|v\|_{0}^{2}
$$

for our special $v$, consequently it follows that $v=0$ and then $w=0$.

(ii) The estimates (5.18) are immediate consequences of (5.10). Concerning (5.19) we only note that by $(5.8)$

$$
\|M(u) f-M(w) f\|_{0} \leq C\|u-w\|_{s_{0}-2}\|f\|_{0}
$$

from which the assertion can easily be derived.

(iii) Fix $v \in H^{s+2}, u \in U_{s}$ and for the time being $w \in H^{s}$. Using the representation (5.14), we get for $|\alpha|=s$

$$
\begin{aligned}
& \left(M(u) D^{\alpha} v, M(u) D^{\alpha} w\right)_{0}= \\
& \quad \sum(-1)^{\left|\alpha_{0}\right|}\left(M(u) D^{\alpha} v, D^{\alpha_{0}} M^{(k)}(u)\left\{D^{\alpha_{1}} u, \ldots, D^{\alpha_{k}} u\right\} D^{\delta} w\right)_{0}
\end{aligned}
$$

with $\left|\alpha_{i}\right| \leq 2,|\delta|=s-2$. Now assume $u_{n} \rightarrow u$ in $U_{s}$, thus $u_{n} \rightarrow u$ in $H^{s^{\prime}}$ with $s \in[0, s)$, and $w_{n} \rightarrow w$ in $H^{s-2}$. According to the above remark, $\left\langle v, w_{n}\right\rangle_{s, u_{n}}$ can essentially be represented as a sum of terms

$$
\sum(-1)^{\left|\alpha_{0}\right|}\left\langle M\left(u_{n}\right) D^{\alpha} v, D^{\alpha_{0}} M^{(k)}\left(u_{n}\right)\left\{D^{\alpha_{1}} u_{n}, \ldots, D^{\alpha_{k}} u_{n}\right\} D^{\delta} w_{n}\right\rangle_{H^{2} \times H^{-2}}
$$


with $\alpha, \alpha_{i}$, and $\delta$ as above, where $\langle\cdot, \cdot\rangle_{H^{2} \times H^{-2}}$ denotes the $L^{2}$-duality map on $H^{2}\left(S, \mathbb{R}^{m}\right) \times$ $H^{-2}\left(S, \mathbb{R}^{m}\right)$. From the smoothness properties of $M$ we conclude

$$
M\left(u_{n}\right) D^{\alpha} v \rightarrow M(u) D^{\alpha} v \text { in } H^{2}\left(S, \mathbb{R}^{m}\right) .
$$

Similarly, uniform boundedness of $\left\|w_{n}\right\|_{s-2}$ and convergence $u_{n} \rightarrow u$ in $H^{s_{0}-2}$ imply via

$$
M^{(k)}\left(u_{n}\right)\left\{D^{\alpha_{1}} u_{n}, \ldots, D^{\alpha_{k}} u_{n}\right\} D^{\delta} w_{n}-M^{(k)}(u)\left\{D^{\alpha_{1}} u, \ldots, D^{\alpha_{k}} u\right\} D^{\delta} w_{n} \rightarrow 0
$$

in $L^{2}$. As strong continuity of linear operators implies weak continuity, we have

$$
M^{(k)}(u)\left\{D^{\alpha_{1}} u, \ldots, D^{\alpha_{k}} u\right\} D^{\delta} w_{n} \rightarrow M^{(k)}(u)\left\{D^{\alpha_{1}} u, \ldots, D^{\alpha_{k}} u\right\} D^{\delta} w
$$

weakly in $L^{2}$, and consequently

$$
D^{\alpha_{0}} M^{(k)}\left(u_{n}\right)\left\{D^{\alpha_{1}} u_{n}, \ldots, D^{\alpha_{k}} u_{n}\right\} D^{\delta} w_{n} \rightarrow D^{\alpha_{0}} M^{(k)}(u)\left\{D^{\alpha_{1}} u, \ldots, D^{\alpha_{k}} u\right\} D^{\delta} w
$$

weakly in $H^{-2}$. Together with (5.20), this completes the proof.

Lemma 5.5. Let $s \geq s_{0}$ be an integer, $u \in \widetilde{U}_{s}$ and assume $\gamma=\rho^{2}$ with $\rho \in C^{\infty}(S)$.

(i) There exists positive constants $c, C$, independent of $u$, such that

$$
\begin{aligned}
\left\|\mathscr{G}^{\prime}(u) v\right\|_{0} & \leq C\left(\|\rho P(u) v\|_{2}+\|v\|_{1}\right), \\
\left(D P(u) v, D^{\prime} \mathscr{G}^{\prime}(u) v\right)_{0} & \leq-c\|\rho P(u) D v\|_{1}^{2}+C\|v\|_{1}^{2}
\end{aligned}
$$

for all $v \in H^{2}\left(S, \mathbb{R}^{m}\right)$ and any derivative $D=D^{\alpha},|\alpha| \leq 1$.

(ii) Moreover, for $|\alpha|=s$ we have

$$
\begin{aligned}
\left\|D^{\alpha} \mathscr{G}(u)\right\|_{-1} & \leq C\left(\left\|\rho P(u) D^{\alpha} u\right\|_{1}+\|u\|_{s}\right), \\
\left(P(u) D^{\alpha} u, D^{\alpha} \mathscr{G}(u)\right)_{0} & \leq-c\left\|\rho P(u) D^{\alpha} u\right\|_{1}^{2}+C\|u\|_{s}^{2} .
\end{aligned}
$$

Proof. (i) To show (5.21), (5.22) it suffices to construct a representation of the form

$$
\mathscr{G}^{\prime}(u) v=\gamma \Delta(u)(P(u) v)+\rho R_{1}(u) v+R_{2}(u) v
$$

with operators $R_{1}(u), R_{2}(u)$ such that

$$
\left\|R_{1}(u) v\right\|_{0},\left\|R_{2}(u) v\right\|_{1} \leq C\|v\|_{1} .
$$

For the part $H^{\prime}(u) v$ of $\mathscr{G}^{\prime}(u) v$, which is a second order differential operator in $v$, this is quite clear using the well-known fact that the linearization of the mean curvature has $\Delta(u)(P(u) v)$ as main part. Concerning $G^{\prime}(u) v$ we note

$$
\begin{aligned}
-G^{\prime}(u) v & =A(u) \Delta^{\prime}(u)\{v\} \gamma+A^{\prime}(u)\{v\} \Delta(u) \gamma, \\
\Delta^{\prime}(u)\{v\} \gamma & =2 \rho \Delta^{\prime}(u)\{v\} \rho+4 \Lambda_{i}^{\prime}(u)\{v\} \rho \Lambda_{i}(u) \rho,
\end{aligned}
$$

hence we have the representation $-G^{\prime}(u) v=\rho R_{1}(u)+R_{2}(u)$ with

$$
\begin{aligned}
R_{1}(u):= & 2 A(u) \Delta^{\prime}(u)\{v\} \rho, \\
R_{2}(u):= & 2\left(A(u)\left(\rho \Delta^{\prime}(u)\{v\} \rho\right)-\rho A(u) \Delta^{\prime}(u)\{v\} \rho\right) \\
& +4 A(u) \Lambda_{i}^{\prime}(u)\{v\} \rho \Lambda_{i}(u) \rho+A^{\prime}(u)\{v\} \Delta(u) \gamma .
\end{aligned}
$$


Due to

$$
\|\Delta(u) \rho\|_{s_{0}-2} \leq C, \quad\left\|\Delta^{\prime}(u)\{v\} \rho\right\|_{-1},\left\|\Lambda_{i}^{\prime}(u)\{v\} \rho\right\|_{0} \leq C\|v\|_{1},
$$

the estimate (5.26) for $R_{1}$ is now a consequence of

$$
\|A(u) f\|_{0} \leq C\|f\|_{-1}, \quad\left\|\Delta^{\prime}(u)\{v\} \rho\right\|_{-1} \leq C\|v\|_{1},
$$

whereas the estimate for $R_{2}$ follows from the commutator estimate (4.33) together with

$$
\|A(u) f\|_{1} \leq C\|f\|_{0}, \quad\left\|A^{\prime}(u)\{v\} f\right\|_{1} \leq C\|v\|_{1}\|f\|_{s_{0}-2} .
$$

(ii) Similar to part (i), it suffices to show the existence of a decomposition

$$
D^{\alpha} \mathscr{G}(u)=\gamma \Delta(u)\left(P(u) D^{\alpha} u\right)+\rho R_{1}(u)+R_{2}(u)
$$

with operators $R_{1}, R_{2}$ allowing the estimates

$$
\left\|R_{1}(u)\right\|_{-1},\left\|R_{2}(u)\right\|_{0} \leq C\|u\|_{s}
$$

Again, for the part $D^{\alpha} H$ of $D^{\alpha} \mathscr{G}$ this is quite clear, where $R_{1}, R_{2}$ are now local differential operators with respect to $u$ of order $s+1$ and $s$, respectively. Concerning $D^{\alpha} G(u)$ we write $\alpha=\beta+\delta$ with $|\beta|=1,|\delta|=s-1$ and calculate

$$
-D^{\alpha} G(u)=2 \rho D^{\beta} A(u) \Delta^{\prime}(u)\left\{D^{\delta} u\right\} \rho+Q_{1}+\ldots+Q_{5}
$$

with

$$
\begin{aligned}
& Q_{1}:=D^{\beta}\left(D^{\delta} A(u) \Delta(u) \gamma-A(u) D^{\delta} \Delta(u) \gamma\right), \\
& Q_{2}:=D^{\beta} A(u)\left(D^{\delta} \Delta(u) \gamma-\Delta^{\prime}(u)\left\{D^{\delta} u\right\} \gamma\right), \\
& Q_{3}:=4 D^{\beta} A(u) \Lambda_{i}^{\prime}(u)\left\{D^{\delta} u\right\} \rho \Lambda_{i}(u) \rho, \\
& Q_{4}:=2 D^{\beta}\left(A(u)\left(\rho \Delta^{\prime}(u)\left\{D^{\delta} u\right\} \rho\right)-\rho A(u) \Delta^{\prime}(u)\left\{D^{\delta} u\right\} \rho\right), \\
& Q_{5}:=2\left(D^{\beta} \rho\right) A(u) \Delta^{\prime}(u)\left\{D^{\delta} u\right\} \rho .
\end{aligned}
$$

Now we set $R_{1}:=2 D^{\beta} A(u) \Delta^{\prime}(u)\left\{D^{\delta} u\right\} \rho$ and $R_{2}:=Q_{1}+\ldots+Q_{5}$. The necessary estimates follow from the properties of $A$, in particular, Proposition 4.7 (ii) and (4.33), and from the additional commutator estimate

$$
\left\|D^{\delta} \Delta(u) \gamma-\Delta^{\prime}(u)\left\{D^{\delta} u\right\} \gamma\right\|_{0} \leq C\|u\|_{s} .
$$

Now we are prepared to formulate and prove the following a-priori estimates for $\mathscr{F}$ w.r. to the bilinear forms $\langle\cdot, \cdot\rangle_{s, u}$.

Proposition 5.6. Let $s \geq s_{0}$ be integer. Then

$$
\begin{aligned}
\left\langle v, \mathscr{F}^{\prime}(u) v\right\rangle_{1, u} & \leq C\|v\|_{1}^{2}, \\
\langle u, \mathscr{F}(u)\rangle_{s, u} & \leq C\|u\|_{s}^{2}
\end{aligned}
$$

for all $u \in \widetilde{U}_{s}$ and $v \in H^{2}\left(S, \mathbb{R}^{m}\right)$ with constants independent of $u$ and $v$.

Proof. We start with the proof of (5.27). Due to (5.21), for any derivative $D$

$$
\left\|D \mathscr{F}^{\prime}(u) v-F^{\prime}(u)\{D v\} \mathscr{G}(u)-F(u)\left(D^{\prime} \mathscr{G}^{\prime}(u) v\right)\right\|_{0} \leq C\left(\|v\|_{1}+\|\rho P(u) v\|_{2}\right)
$$


and consequently by Lemma 5.1

$$
D \mathscr{F}^{\prime}(u) v=F(u)\left(\mathscr{F}(u) \cdot \Lambda(u)(P(u) D v)+D^{G^{\prime}}(u) v\right)+R(u) v,
$$

where the remainder term satisfies

$$
\|R(u) v\|_{0} \leq C\left(\|v\|_{1}+\|\rho P(u) v\|_{2}\right) .
$$

Further, by (5.21) we have

$$
\left\|D^{\prime} \mathscr{G}^{\prime}(u) v\right\|_{-1} \leq C\left(\|v\|_{1}+\|\rho P(u) v\|_{2}\right),
$$

and moreover

$$
\|\mathscr{F}(u) \cdot \Lambda(u)(P(u) D v)\|_{-1} \leq C\|v\|_{1} .
$$

Hence by Lemma 5.2 it follows that

$$
\left\langle D v, D \mathscr{F}^{\prime}(u) v\right\rangle_{0, u} \leq\left(P(u) D v, \mathscr{F}(u) \cdot \Lambda(u)(P(u) D v)+D \mathscr{G}^{\prime}(u) v\right)_{0}+I(u) v^{2}
$$

where now

$$
I(u) v^{2} \leq C\left(\|v\|_{1}+\|\rho P(u) v\|_{2}\right)\|v\|_{1}
$$

Writing

$$
\begin{aligned}
(P(u) D v, & \mathscr{F}(u) \cdot \Lambda(u)(P(u) D v))_{0} \\
& =\frac{1}{2} \int_{S}\left(\Lambda_{i}(u)\left(\mathscr{F}_{i}(u)(P(u) D v)^{2}\right)-(P(u) D v)^{2} \Lambda_{i}(u) \mathscr{F}_{i}(u)\right) d S,
\end{aligned}
$$

an integration by parts on $S$ using (5.5) yields

$$
\left|(P(u) D v, \mathscr{F}(u) \cdot \Lambda(u)(P(u) D v))_{0}\right| \leq C\|v\|_{1}^{2},
$$

hence together with Lemma 5.5, (5.22) we obtain the estimate (5.27).

Further, to prove (5.28) we use the abbreviation

$$
\|u\|_{s+1}^{\prime}:=\left(\|u\|_{s}+\sum_{|\alpha|=s}\left\|\rho P(u) D^{\alpha} u\right\|_{1}\right) .
$$

Using Proposition 4.6, (ii) we write

$$
D^{\alpha} \mathscr{F}(u)=F(u) D^{\alpha} \mathscr{G}(u)+F^{\prime}(u)\left\{D^{\alpha} u\right\} \mathscr{G}(u)+R_{1}(u),
$$

where $R_{1}$ allows the estimate

$$
\left\|R_{1}(u)\right\|_{0} \leq C\left(\|u\|_{s}\|\mathscr{G}(u)\|_{s_{0}-2}+\|\mathscr{G}(u)\|_{s-1}\right) \leq C\|u\|_{s+1}^{\prime}
$$

because of

$$
\|\mathscr{G}(u)\|_{s-1} \leq C\left(\sum_{|\alpha|=s}\left\|D^{\alpha} \mathscr{G}(u)\right\|_{-1}+\|\mathscr{G}(u)\|_{0}\right)
$$

and (5.23). Further, using Lemma 5.1 we have

$$
D^{\alpha} \mathscr{F}(u)=F(u)\left(D^{\alpha} \mathscr{G}(u)+\mathscr{F}(u) \cdot \Lambda(u)\left(P(u) D^{\alpha} u\right)\right)+R_{1}(u)+R_{2}(u),
$$

where again

$$
\left\|R_{2}(u)\right\|_{0} \leq C\left\|D^{\alpha} u\right\|_{0} \leq C\|u\|_{s}
$$


and consequently

$$
\left\langle R_{1}(u)+R_{2}(u), D^{\alpha} u\right\rangle_{0, u} \leq C\|u\|_{s+1}^{\prime}\|u\|_{s} .
$$

By Lemma 5.2, (5.11) we obtain

$$
\left\langle D^{\alpha} u, D^{\alpha} \mathscr{F}(u)\right\rangle_{0, u}=\left(P(u) D^{\alpha} u, \mathscr{F}(u) \cdot \Lambda(u)\left(P(u) D^{\alpha} u\right)+D^{\alpha} \mathscr{G}(u)\right)_{0}+I(u),
$$

where $I(u)$ allows the estimate

$$
\begin{aligned}
I(u) & \leq C\left\|D^{\alpha} u\right\|_{0}\left(\left\|D^{\alpha} \mathscr{G}(u)\right\|_{-1}+\left\|\mathscr{F}(u) \cdot \Lambda(u)\left(P(u) D^{\alpha} u\right)\right\|_{-1}\right) \\
& \leq C\left(1+\|u\|_{s+1}^{\prime}\right)\|u\|_{s} .
\end{aligned}
$$

Finally, by an integration by parts as above we get

$$
\left(D^{\alpha} u, \mathscr{F}(u) \cdot \Lambda(u)\left(P(u) D^{\alpha} u\right)_{0} \leq C\|u\|_{s}^{2} .\right.
$$

Together with (5.24), this completes the proof.

The structure of $F^{\prime}(u)$ as stated in Lemma 5.1 and the integration by parts argument used in the above proof are necessary to cover the case of a $\gamma$ which can degenerate. If $\gamma$ is strictly positive the argumentation can be simplified by using Lemma 5.1 to obtain the estimate

$$
\left\|F^{\prime}(u)\{v\} f\right\|_{0} \leq C\left(\|P(u) v\|_{1}+\|v\|_{0}\right)\|f\|_{s_{0}-1} .
$$

To conclude this section we add some remarks about the case of a slip factor $\delta$ (introduced in (2.8)) different from one. The nonlinear operator of the evolution equation is now

$$
\mathscr{F}_{1}(u):=F_{1}(u)\left(\mathscr{G}_{1}(u)\right)
$$

with

$$
F_{1}(u) f:=(\delta i d+(1-\delta) N(u) P(u)) F(u) f, \quad \mathscr{G}_{1}(u)=H(u)+\delta G(u) .
$$

Clearly, Lemma 4.5 and Proposition 4.6 continue to hold also for $F_{1}$. To see that $F_{1}^{\prime}(u)$ satisfies an estimate parallel to (5.29) as well, note that due to (5.6) we have

$$
\begin{aligned}
\left\|P^{\prime}(u)\{v\} w\right\|_{0} \leq C\left(\|P(u) v\|_{1}+\|v\|_{0}\right)\|w\|_{s_{0}-2}, \\
\left\|N^{\prime}(u)\{v\} z\right\|_{0} \leq C\left(\|P(u) v\|_{1}+\|v\|_{0}\right)\|z\|_{s_{0}-2} .
\end{aligned}
$$

This implies such an estimate for $F_{1}$. Hence, by changing the definition (5.7) of $M$ into

$$
M(u) v:=v-\delta \Lambda(u)(\psi(u) P(u) v)
$$

and $\langle\cdot, \cdot\rangle_{s, u}$ accordingly, we obtain the crucial estimates (5.27), (5.28) of Proposition 5.6 also for $\mathscr{F}_{1}$, at least in the case of strictly positive $\gamma$. Note that for $\delta=0$ the bilinear forms $\langle\cdot, \cdot\rangle_{s, u}$ are in fact independent of $u$.

\section{Proof of Theorems 3.1 and 3.2}

Lemma 6.1. Fix $\widetilde{U}_{s_{0}} \subset U_{s_{0}}$. Let $u, v \in C_{w}\left([0, T], H^{s_{0}}\right) \cap C_{w}^{1}\left([0, T], H^{s_{0}-2}\right)$ be two solutions of (3.2) with

$$
u(t), v(t) \in \widetilde{U}_{s_{0}} \text { for } t \in[0, T] .
$$


There exists a real number $C$ depending only on $T$ and $\widetilde{U}_{s_{0}}$ such that

$$
\|u(t)-v(t)\|_{1} \leq C\|u(0)-v(0)\|_{1} \text { for all } t \in[0, T] .
$$

Proof. We put $w(t):=v(t)-u(t)$ and remark

$$
u, v \in C\left([0, T], H^{s}\right) \cap C^{1}\left([0, T], H^{s-2}\right) \text { for } 2 \leq s<s_{0} ;
$$

in particular, the mapping $[0, T] \ni t \mapsto\langle w(t), w(t)\rangle_{0, u(t)}$ is differentiable and we will show

$$
\frac{d}{d t}\langle w(t), w(t)\rangle_{1, u(t)} \leq C\langle w(t), w(t)\rangle_{1, u(t)},
$$

which implies (6.1) via Gronwall's lemma. Recalling that $H$ is a quasilinear second order differential operator, we have

$$
\left\|H^{\prime}(z) w\right\|_{1} \leq C\|w\|_{3}, \quad\left\|H^{\prime \prime}(z)\{w, w\}\right\|_{1} \leq C\|w\|_{3}\|w\|_{s_{0}-2}
$$

and accordingly by (4.32)

$$
\left\|G^{\prime}(z) w\right\|_{1} \leq C\|v\|_{3}, \quad\left\|G^{\prime \prime}(z)\{w, w\}\right\|_{1} \leq C\|w\|_{3}\|w\|_{s_{0}-2} .
$$

Consequently, together with Lemma 4.5, (4.20) we obtain

$$
\left\|\mathscr{F}^{\prime \prime}(z)\{w, w\}\right\|_{1} \leq C\|w\|_{3}\|w\|_{s_{0}-2} .
$$

Using Taylor's theorem we have

$$
w^{\prime}(t):=\frac{d}{d t} w(t)=\mathscr{F}(v(t))-\mathscr{F}(u(t))=\mathscr{F}^{\prime}(u(t)) w(t)+R(u(t), v(t)) ;
$$

the remainder term therein can be estimated by (6.3) and norm convexity

$$
\|R(u(t), v(t))\|_{1} \leq C_{1}\|w(t)\|_{s_{0}-2}\|w(t)\|_{3} \leq C_{2}\|w(t)\|_{s_{0}}\|w(t)\|_{1} \leq C_{3}\|w(t)\|_{1} .
$$

Thus, together with (5.27), we obtain

$$
\left\langle w(t), w^{\prime}(t)\right\rangle_{1, u(t)}=\left\langle w(t), \mathscr{F}^{\prime}(u(t)) w(t)+R(u(t), v(t))\right\rangle_{1, u(t)} \leq C\|w(t)\|_{1}^{2} .
$$

Furthermore, recalling (5.8), we have

$$
\left\|M^{\prime}(u(t))\left\{u^{\prime}(t)\right\} w(t)\right\|_{1} \leq C_{2}\left\|u^{\prime}(t)\right\|_{s_{0}-2}\|w(t)\|_{1},
$$

hence

$$
\left\|u^{\prime}(t)\right\|_{s_{0}-2}=\|\mathscr{F}(u(t))\|_{s_{0}-2} \leq C
$$

gives

$$
\left\|M^{\prime}(u(t))\left\{u^{\prime}(t)\right\} w(t)\right\|_{1} \leq C\|w(t)\|_{1} .
$$

Consequently, considering

$$
\begin{aligned}
\frac{1}{2} \frac{d}{d t}\langle w(t) & , w(t)\rangle_{1, u(t)} \\
& =\left\langle w(t), w^{\prime}(t)\right\rangle_{1, u(t)}+\left(M(u(t)) w(t), M^{\prime}(u(t))\left\{u^{\prime}(t)\right\} w(t)\right)_{1},
\end{aligned}
$$

we obtain the desired estimate (6.1) from (6.4), (6.5).

We note a result on nonlinear interpolation, whose proof can be found in [2], Propostion A.1 and Remark A.2. 
Lemma 6.2. Let $\mathscr{U} \subseteq H^{s}\left(S, \mathbb{R}^{m}\right), s \geq 1$ be an open set. Let $T_{\alpha}: \mathscr{U} \rightarrow H^{1}\left(S, \mathbb{R}^{m}\right)$ be mappings with $T_{\alpha}\left(\mathscr{U} \cap H^{s+1}\right) \subseteq H^{s+1} ; \alpha$ runs through a certain index set $I$. Further, assume Lipschitz continuity of $T_{\alpha}$ in $H^{1}$ and boundedness of $T_{\alpha}$ in $H^{s+1}$ :

$$
\begin{gathered}
\left\|T_{\alpha}(u)-T_{\alpha}(v)\right\|_{1} \leq C\|u-v\|_{1} \text { for all } u, v \in \mathscr{U}, \\
\left\|T_{\alpha}(u)\right\|_{s+1} \leq C\left(1+\|u\|_{s+1}\right) \text { for all } u \in \mathscr{U} \cap H^{s+1}
\end{gathered}
$$

with a constant $C$ independent of $u, v$ and $\alpha \in I$. Then $T_{\alpha}\left(\mathscr{U} \cap H^{s}\right) \subseteq H^{s}$ and the mappings $T_{\alpha}: \mathscr{U} \subseteq H^{s} \rightarrow H^{s}$ are continuous, uniformly with respect to $\alpha \in I$.

Now we are prepared for the proof of our theorems. In many respects, it is parallel to the proof of the main results in [9].

Proof of Theorem 3.1. Step 1. We show that for any given $\bar{u}_{0} \in U_{s_{0}}$ and any integer $s \geq s_{0}$ there exist $T=T\left(\bar{u}_{0}, s\right)>0$ and $\delta=\delta\left(\bar{u}_{0}, s\right)>0$ such that the Cauchy problem (3.2) has a unique solution in the class

$$
u \in C_{w}\left([0, T], U_{s}\right) \cap C_{w}^{1}\left([0, T], H^{s-2}\right)
$$

for all initial values $u_{0} \in H^{s}$ with $\left\|u_{0}-\bar{u}_{0}\right\|_{s_{0}} \leq \delta$. The uniqueness of the solution follows immediately from Lemma 6.1. In order to prove the existence we use Theorem 3.4. With a fixed $s \geq s_{0}$ and an $\varepsilon \in(0,1]$ which will be fixed below, we put

$$
\begin{aligned}
& X=H^{s+2}\left(S, \mathbb{R}^{m}\right), \quad\|\cdot\|_{X}=\|\cdot\|_{s_{0}+2}+\varepsilon\|\cdot\|_{s+2} ; \\
& Y=H^{s}\left(S, \mathbb{R}^{m}\right), \quad\|\cdot\|_{Y}=\|\cdot\|_{s_{0}}+\varepsilon\|\cdot\|_{s} ; \\
& Z=H^{s-2}\left(S, \mathbb{R}^{m}\right), \quad\|\cdot\|_{Z}=\|\cdot\|_{s_{0}-2}+\varepsilon\|\cdot\|_{s-2} .
\end{aligned}
$$

Further, let $\widetilde{U}_{s}$ be as in Section 5 and assume that the given $\bar{u}_{0}$ is an interior point. Then, according to the results of Section 5 , for $u \in \widetilde{U}_{s}$ the bilinear forms $\langle v, w\rangle_{s, u}^{\varepsilon}: X \times Z \rightarrow \mathbb{R}$ satisfy the requirements $(\mathrm{H})$ of Section 3 ; note that the constants $C, M$ in $(\mathrm{H})$ can be chosen independently of $\varepsilon$. As in the proof of Theorem 3.3 we choose $w_{0} \in C^{\infty}\left(S, \mathbb{R}^{m}\right)$ and $R>0$ (both independent of $\varepsilon$ ) such that

$$
\left\|w_{0}-u_{0}\right\|_{s_{0}} \leq R /\left(32 C^{5}\right)^{1 / 2}, \quad\left\{w_{0}+v \mid v \in B\right\} \subseteq \widetilde{U}_{s_{0}}
$$

with the ball $B:=\left\{v \in Y \mid\|v\|_{Y}<R\right\}$. We set

$$
\langle v, w\rangle_{u}:=\langle v, w\rangle_{s, w_{0}+u}^{\varepsilon}, \quad\|v\| \mid=\langle v, v\rangle_{w_{0}+v}
$$

and define a map $\mathscr{H}: B \subseteq Y \rightarrow Z$ by

$$
\mathscr{H}(v):=\mathscr{F}\left(v+w_{0}\right), \quad u \in B .
$$

Further, the mapping $\mathscr{H}: B \subseteq Y \rightarrow Z$ is weakly sequentially continuous and

$$
\left\langle w_{0}+v, \mathscr{H}(v)\right\rangle \leq C_{1}\left\|w_{0}+v\right\|_{Y}^{2} \leq C_{2}
$$

by Proposition 5.6. Moreover we have

$$
\|\mathscr{H}(v)\|_{Z} \leq C_{3}\left\|v+w_{0}\right\|_{Y} \leq C_{4}
$$

and

$$
\left|\left\langle w_{0}, \mathscr{H}(v)\right\rangle_{v}\right| \leq C_{5}\left\|w_{0}\right\|_{X}\|\mathscr{H}(v)\|_{Z} \leq C_{6} .
$$


These estimates hold for all $v \in B$ with constants $C_{1}, \ldots$ which may depend on $C, M, R, s$ and $\bar{u}_{0}, w_{0}$, but not on $v$. Gathering them, we obtain the inequality

$$
2\langle v, \mathscr{H}(v)\rangle_{v}+M\|\mathscr{H}(v)\|_{Z}\|v\| \leq C_{7} \text { for all } v \in B \cap X .
$$

Now, let $u_{0} \in H^{s}\left(S, \mathbb{R}^{m}\right)$ be given such that

$$
\left\|u_{0}-\bar{u}_{0}\right\|_{s_{0}} \leq R /\left(32 C^{5}\right)^{1 / 2}
$$

Hence, with $r:=R /\left(2 C^{3}\right)^{1 / 2}$ we find

$$
\left\|u_{0}-w_{0}\right\| \mid \leq C\left(\left\|u_{0}-\bar{u}_{0}\right\|_{s_{0}}+\left\|\bar{u}_{0}-w_{0}\right\|_{s_{0}}+\varepsilon\left(\left\|u_{0}\right\|_{s}+\left\|w_{0}\right\|_{s}\right)\right) \leq r
$$

if $\varepsilon$ is chosen according to

$$
\varepsilon:=\min \left\{1, r / 4 C\left(\left\|u_{0}\right\|_{s}+\left\|w_{0}\right\|_{s}\right)\right\} .
$$

By Theorem 3.4, applied to $\mathscr{H}$, there exists $T>0$, independent of $u_{0}$ with (6.6), and a solution

$$
v \in C_{w}\left([0, T], B \cap H^{s}\right) \cap C_{w}^{1}\left([0, T], H^{s-2}\right)
$$

of

$$
d v(t) / d t=\mathscr{G}(v(t)) \text { for } t \in[0, T], \quad v(0)=u_{0}-w_{0} .
$$

Then $u:=v+w_{0}$ is a solution of (3.2) with initial value $u(0)=u_{0}$ and we have

$$
\|u(t)\|_{s} \leq\left\|w_{0}\right\|_{s}+\|v(t)\|_{s} \leq\left\|w_{0}\right\|_{s}+\varepsilon^{-1}\|v(t)\|_{Y},
$$

which in view of (6.7) implies

$$
\|u(t)\|_{s} \leq C\left(1+\|u(0)\|_{s}\right) .
$$

Step 2. Let $u, \tilde{u}$ be two solutions of $(3.2)$ in $[0, T]$ according to Step 1 with initial values

$$
u(0), \tilde{u}(0) \in \mathscr{U}, \quad \mathscr{U}:=\left\{v \in H^{s} \mid\left\|v-\bar{u}_{0}\right\|_{s_{0}} \leq \delta\right\},
$$

$\delta>0$ sufficiently small. Lemma 6.1 gives

$$
\|u(t)-\tilde{u}(t)\|_{1} \leq C\|u(0)-\tilde{u}(0)\|_{1} .
$$

For fixed $t \in[0, T]$ we consider the evolution operator

$$
\mathscr{U} \ni u_{0} \mapsto T_{t}\left(u_{0}\right):=u(t) \in H^{s}
$$

assigning to any initial value $u_{0}$ the value of the corresponding solution of (3.2) at time $t$. By Step 1 with $s$ replaced by $s+1$ we obtain $T_{t}\left(\mathscr{U} \cap H^{s+1}\right) \subseteq H^{s+1}$ and the estimate

$$
\left\|T_{t}\left(u_{0}\right)\right\|_{s+1} \leq C\left(1+\left\|u_{0}\right\|_{s+1}\right) .
$$

(6.9), (6.10) and together with the interpolation result from Lemma 6.2 shows the continuity of the mapping

$$
\mathscr{U} \cap H^{s} \ni u_{0} \mapsto u(t) \in H^{s} \text { for } s \geq s_{0},
$$

uniformly with respect to $t \in[0, T]$.

Step 3. To complete the proof of Theorem 3.1 it remains to show that the solutions according to Step 1 actually belong to

$$
u \in C\left([0, T], H^{s}\right) \cap C^{1}\left([0, T], H^{s-2}\right) .
$$


To do this, we approximate the initial value $u_{0}=u(0)$ by a sequence $u_{0}^{n} \in H^{s+1}$ such that $u_{0}^{n} \rightarrow u_{0}$ in $H^{s}$. Then by Step 1, for $n$ sufficiently large, there exist solutions $u_{n}$ of (3.2) with $u_{n}(0)=u_{0}^{n}$ in the class

$$
u_{n} \in C_{w}\left([0, T], H^{s+1}\right) \cap C_{w}^{1}\left([0, T], H^{s-1}\right),
$$

which in particular implies

$$
u_{n} \in C\left([0, T], H^{s}\right) \cap C^{1}\left([0, T], H^{s-2}\right) .
$$

On the other hand, by Step 2, we have $u_{n}(t) \rightarrow u(t)$ in $H^{s}$ uniformly with respect to $t \in[0, T]$. As the uniform limit of continuous functions is continuous again, this implies (6.11).

Proof of Theorem 3.2. Let a solution $u \in C\left([0, T], U_{s}\right) \cap C^{1}\left([0, T], H^{s-2}\right)$ be given. The set $\{u(t) \mid t \in[0, T]\}$ is compact in $H^{s}$ and can be covered by the open sets $\{v \in$ $\left.H^{s} \mid\|v-u(t)\|_{s}<\delta(u(t), s+1)\right\}, t \in[0, T]$, where $\delta(u(t), s+1)$ are the same as in the proof of Theorem 3.1. Choosing a finite subcover, we find from this theorem and the autonomous character of (3.2) that there is a $T_{0}>0$ such that for any $t \in[0, T]$ with $u(t) \in H^{s+1}$, we have

$$
\left.u\right|_{\left[t, T_{1}\right]} \in C\left(\left[t, T_{1}\right], U_{s+1}\right) \cap C^{1}\left(\left[t, T_{1}\right], H^{s-1}\right), \quad T_{1}:=\min \left\{t+T_{0}, T\right\} .
$$

Proceeding stepwise, we obtain (i).

A similar compactness argument together with Theorem 3.1 and its proof ensures the existence of $T_{2}>0$ such that the following is true for all $t \in[0, T]$ : Problem (3.2) is solvable on the time interval $\left[0, T_{2}\right]$ (in the class (3.13)) for all initial values $z$ sufficiently near $u(t)$, and the mapping which assigns to $z$ its corresponding solution $V(\cdot, z)$ is continuous with values in $C\left(\left[0, T_{2}\right], H^{s}\right)$. We choose $t_{i} \in[0, T]$ such that $0=t_{0}<\ldots<t_{n}=T$, $t_{i}-t_{i-1}<T_{2}$, and open $H^{s}$-neighbourhoods $K_{i}$ of $u\left(t_{i}\right)$ small enough to ensure that $V$ is defined on $K_{i}$ and $V\left(t_{i}-t_{i-1}, K_{i-1}\right) \subset K_{i}, i=n-1, \ldots, 1$. Now (ii) follows from the continuity of the composition of continuous maps.

\section{A. Proof of Theorem 3.4}

We will construct a solution of (3.19) by implicit time discretization, solving the nonlinear problems in each timestep by Galerkin approximations. For this purpose, we need the following lemma:

Lemma A.1. For any $K \in\left(0, r^{2}\right)$ there is an $\varepsilon_{0}>0$ such that for any $\varepsilon \in\left(0, \varepsilon_{0}\right]$ and any $v \in Y$ satisfying $\|v\|^{2} \leq K$ there is a $u^{*} \in B$ satisfying

$$
u^{*}=v+\varepsilon \mathscr{G}\left(u^{*}\right)
$$

and the estimate

$$
\left\|u^{*}||^{2} \leq\left|\|v \mid\|^{2}+\varepsilon \beta\left(\left\|u^{*}\right\|^{2}\right) \leq 2 K .\right.\right.
$$


Proof: For arbitrary $v \in Y, u \in X \cap B$ we have

$$
\begin{aligned}
\langle u, u-\varepsilon \mathscr{G}(u)-v\rangle_{u} & =\|u\| \|^{2}-\varepsilon\langle u, \mathscr{G}(u)\rangle_{u}-(u, v)_{u} \\
& \geq\|u\|^{2}-\frac{\varepsilon}{2} \beta\left(\|u\|^{2}\right)+\frac{\varepsilon M}{2}\|\mathscr{G}(u)\|_{Z}\|u\|^{2}-\|u\|\|v\|_{u} \\
& \geq \frac{1}{2}\left(\|u\|^{2}-\varepsilon \beta\left(\|u\|^{2}\right)-\|v\|_{u}^{2}+\varepsilon M\|\mathscr{G}(u)\| Z\|u\|^{2}\right) .
\end{aligned}
$$

Choose $\varepsilon_{0}>0$ such that for all $\varepsilon \in\left(0, \varepsilon_{0}\right]$ and for all $s \in\left[0,2 C^{4} K\right]$

$$
\begin{aligned}
K-\varepsilon \beta(s) & \geq 0, \\
1-\varepsilon \beta^{\prime}(s) & \geq 0 .
\end{aligned}
$$

Assume now $v \in B,\|v v\|^{2} \leq K$. Let

$$
\mathscr{B}:=\left\{u \in Y \mid\|u\|_{Y}^{2} \leq 2 K C^{3}\right\}
$$

and note that $\mathscr{B}$ is a closed convex subset of $B$. Assume $\|u\|_{Y}^{2}=2 K C^{3}$. Then

$$
\begin{gathered}
2 C^{2} K=C^{-1}\|u\|_{Y}^{2} \leq\|\| u\left\|^{2} \leq C\right\| u \|_{Y}^{2}=2 C^{4} K, \\
\|v\|_{u}^{2} \leq C\|v\|_{Y}^{2} \leq C^{2}\|v\| \|^{2} \leq C^{2} K .
\end{gathered}
$$

Therefore, for $\varepsilon \in\left(0, \varepsilon_{0}\right]$,

$$
\langle u, u-\varepsilon \mathscr{G}(u)-v\rangle_{u} \geq \frac{1}{2}\left(C^{2} K-\varepsilon \beta\left(\|u\|^{2}\right)\right) \geq 0 .
$$

Let $\left\{M_{n}\right\}$ be an increasing sequence of finite-dimensional subspaces of $X$ whose union is dense in $X$. We fix $n$, choose a basis $\left\{e_{1}, \ldots, e_{n}\right\}$ of $M_{n}$ and show that the variational equality

$$
\left\langle w, u_{n}-\varepsilon \mathscr{G}\left(u_{n}\right)-v\right\rangle_{u}=0 \text { for all } w \in M_{n}
$$

has a solution $u_{n} \in M_{n} \cap \mathscr{B}$. Note that (A.7) is equivalent to $g\left(u_{n}\right)=0$ where $g$ : $M_{n} \cap \mathscr{B} \rightarrow M_{n}$ is defined by

$$
g(u):=P_{u}\left(u-\varepsilon^{\mathscr{G}}(u)-v\right) \quad \text { with } \quad P_{u}(z):=\sum_{i=1}^{n}\left\langle e_{i}, z\right\rangle_{u} e_{i} .
$$

Due to (H4), $g$ is continuous. Assume now $g(u) \neq 0$ for all $u \in M_{n} \cap \mathscr{B}$. Then we define the continuous operator $f: M_{n} \cap \mathscr{B} \rightarrow M_{n}$ by

$$
f(u):=-\sqrt{2 K C^{3}} g(u) /\|g(u)\|_{Y} .
$$

As $\|f(u)\|_{Y}^{2}=2 K C^{3}, f$ maps the closed convex set $M_{n} \cap \mathscr{B}$ into itself. Therefore, by Brouwer's fixed point theorem, there is an $\bar{u} \in M_{n} \cap \mathscr{B}$ such that $\bar{u}=f(\bar{u})$. Consequently, $\|\bar{u}\|_{Y}^{2}=2 K C^{3}$, and from (A.6) we obtain the contradictory inequality

$$
\begin{aligned}
0 & <\|\bar{u}\|^{2}=\langle\bar{u}, f(\bar{u})\rangle_{\bar{u}}=-\frac{\sqrt{2 K C^{3}}}{\|g(u)\|_{Y}}\langle\bar{u}, g(\bar{u})\rangle_{\bar{u}} \\
& =-\frac{\sqrt{2 K C^{3}}}{\|g(u)\|_{Y}}\langle\bar{u}, \bar{u}-\varepsilon \mathscr{G}(\bar{u})-v\rangle_{\bar{u}} \leq 0 .
\end{aligned}
$$

Therefore, (A.7) is solvable for every $n$, and as $\left\{u_{n}\right\}$ is bounded in $Y$, we can assume 
without loss of generality that $u_{n} \rightarrow u^{*}$ in $Y$ for some $u^{*} \in \mathscr{B}$. Passage to the limit in (A.7) yields by (H4)

$$
\left\langle w, u^{*}-\varepsilon \mathscr{G}\left(u^{*}\right)-v\right\rangle_{u^{*}}=0 \text { for all } w \in M_{n}, n=1,2, \ldots
$$

and consequently by the density assumption

$$
\left\langle w, u^{*}-\varepsilon \mathscr{G}\left(u^{*}\right)-v\right\rangle_{u^{*}}=0 \text { for all } w \in X .
$$

The nondegeneracy of $\langle\cdot, \cdot\rangle_{u^{*}}$ yields (A.1). To show the estimate (A.2), note at first that

$$
\left\|u^{*}\right\|^{2} \leq \underset{n \rightarrow \infty}{\lim _{n \rightarrow \infty}}\left\|u_{n}\right\|^{2} \leq 2 C^{4} K .
$$

Thus, the second inequality in (A.2) follows from (A.4). To show the first inequality we assume without loss of generality $\|v\|\|\leq\| u^{*} \|$ and use (A.5), (A.3), and (H4) to obtain

$$
\begin{aligned}
\left\|u^{*}\right\|^{2} & -\varepsilon \beta\left(\left\|u^{*}\right\|^{2}\right) \leq \varliminf_{n \rightarrow \infty}\left(\left\|u_{n}\right\|^{2}-\varepsilon \beta\left(\left\|u_{n}\right\|^{2}\right)\right) \\
& \leq \underset{n \rightarrow \infty}{\lim }\left(\|v\|_{u_{n}}^{2}-M \varepsilon\left\|\mathscr{G}\left(u_{n}\right)\right\|_{Z}\left\|u_{n}\right\|^{2}\right) \leq\|v\|_{u^{*}}^{2}-M \varepsilon\left\|\mathscr{G}\left(u^{*}\right)\right\|_{Z}\left\|u^{*}\right\|^{2} \\
& \leq\|v\|^{2}+M\left\|u^{*}-v\right\|_{Z}\|v\|\left\|^{2}-M \varepsilon\right\| \mathscr{G}\left(u^{*}\right)\left\|_{Z}\right\| u^{*} \|^{2} \\
& =\|v\|^{2}+M \varepsilon\left\|\mathscr{G}\left(u^{*}\right)\right\|_{Z}\|v\|^{2}-M \varepsilon\left\|\mathscr{G}\left(u^{*}\right)\right\|_{Z}\left\|u^{*}\right\|^{2} \leq\|v\|^{2} . \quad \square
\end{aligned}
$$

As a further preparation for the proof of Theorem 3.4 we need the following simple result on approximate solutions of the ordinary differential equation (3.18).

Lemma A.2. Assume $u_{0} \in B$ and let $\rho \in C^{1}[0, T]$ be the solution of (3.18). There is an $n_{0} \in \mathbb{N}$ such that for $n \geq n_{0}$ and $k=1, \ldots, n$ there are $\rho_{n}^{k}, r_{n} \in \mathbb{R}$ such that

$$
\rho_{n}^{0}=\left\|u_{0}\right\|^{2}, \quad \rho_{n}^{k}+\delta_{n} \beta\left(\rho_{n}^{k+1}\right) \leq \rho_{n}^{k+1} \leq \rho\left((k+1) \delta_{n}\right)+r_{n}, \quad r_{n} \rightarrow 0
$$

where $\delta_{n}:=T / n$.

Proof. If $n_{0}$ is sufficiently large, $n \geq n_{0}$, there exist solutions $\rho_{n} \in C^{1}[0, T]$ to the initial value problems

$$
\rho_{n}^{\prime}(t)=\beta\left(\rho_{n}(t)\right)+1 / \sqrt{n}, \quad \rho_{n}(0)=\left\|u_{0}\right\|^{2} .
$$

We set

$$
\rho_{n}^{k}:=\rho_{n}\left(k \delta_{n}\right), \quad k=0, \ldots, n .
$$

Then

$$
\rho_{n}^{k+1}-\rho_{n}^{k}=\delta_{n} \rho_{n}^{\prime}(\xi)=\delta_{n} \beta\left(\rho_{n}(\xi)\right)+\delta_{n} n^{-1 / 2}
$$

for some $\xi \in\left(k \delta_{n},(k+1) \delta_{n}\right)$. Moreover,

$$
\left|\beta\left(\rho_{n}(\xi)\right)-\beta\left(\rho_{n}^{k+1}\right)\right| \leq S\left|\rho_{n}(\xi)-\rho_{n}^{k+1}\right| \leq S^{\prime} n^{-1}
$$

with constants $S, S^{\prime}$ independent of $n$. Thus

$$
\rho_{n}^{k+1}-\rho_{n}^{k} \geq \delta_{n} \beta\left(\rho_{n}^{k+1}\right)+\delta_{n} n^{-1 / 2}-S^{\prime} \delta_{n} n^{-1} \geq \delta_{n} \beta\left(\rho_{n}^{k+1}\right)
$$

for $n \geq n_{0}, n_{0}$ sufficiently large. Moreover, well-known results on the dependence of the solution of ODE's on their right hand side ensure

$$
r_{n}:=\max _{t \in[0, T]}\left|\rho_{n}(t)-\rho(t)\right| \rightarrow 0, \quad n \rightarrow \infty,
$$


hence

$$
\rho_{n}(t) \leq \rho(t)+r_{n}, \quad t \in[0, T] .
$$

This proves the lemma.

Proof of Theorem 3.4. In a first step, we construct approximations $u_{n}^{k}$ for the solution at time $k T / n$. Choose $K \in\left(\max _{t \in[0, T]} \rho(t), r^{2}\right)$ and choose $\varepsilon_{0}>0$ such that the assertions of Lemma A.1 and (A.5) hold. Let $n_{0} \in \mathbb{N}$ be at least as large as in Lemma A.2 and assume additionally $n_{0} \geq T / \varepsilon_{0}$ and

$$
\rho(t)+r_{n} \leq K \text { for } n \geq n_{0} \text { and } t \in[0, T] .
$$

Now we fix $n \geq n_{0}$ and show the existence of $u_{n}^{k} \in B, k=0, \ldots, n$ such that

$$
\begin{aligned}
u_{n}^{k+1} & =u_{n}^{k}+\delta_{n} \mathscr{G}\left(u_{n}^{k+1}\right), \quad k=0, \ldots, n-1, \\
u_{n}^{0} & =u_{0}, \\
\left\|u_{n}^{k}\right\|^{2} & \leq \rho_{n}^{k}
\end{aligned}
$$

where the $\rho_{n}^{k}$ are given by Lemma A.2. For $k=0$, existence and the estimate are clear. Assume now $u_{n}^{0}, \ldots, u_{n}^{k}$ are constructed according to these conditions for $0 \leq k \leq n-1$. Our assumptions imply $\delta_{n} \leq \varepsilon_{0}$ and $\left\|u_{n}^{k}\right\|^{2} \leq K$, hence the existence of $u_{n}^{k+1}$ follows from Lemma A.1. Moreover, by (A.2), $\left\|u_{n}^{k+1}\right\|^{2} \leq 2 K$ and

$$
\left\|u_{n}^{k+1}\right\|^{2} \leq\left\|u_{n}^{k}\right\|^{2}+\delta_{n} \beta\left(\left\|u_{n}^{k+1}\right\|^{2}\right) \leq \rho_{n}^{k}+\delta_{n} \beta\left(\left\|u_{n}^{k+1} \mid\right\|^{2}\right),
$$

hence

$$
\left\|u_{n}^{k+1}\right\|^{2}-\delta_{n} \beta\left(\left\|u_{n}^{k+1} \mid\right\|^{2}\right) \leq \rho_{n}^{k+1}-\delta_{n} \beta\left(\rho_{n}^{k+1}\right) .
$$

Note that (A.5) implies that the mapping $s \mapsto s-\delta_{n} \beta(s)$ is monotone increasing on $[0,2 K]$, hence $\left\|u_{n}^{k+1}\right\|^{2} \leq \rho_{n}^{k+1}$.

In a second step, we approximate $u$ on $[0, T]$ by piecewise linear functions $u_{n}$ and piecewise constant functions $\bar{u}_{n}, n \geq n_{0}$, given by

$$
\begin{aligned}
u_{n}(t):= & u_{n}^{k}+\delta_{n}^{-1}\left(t-k \delta_{n}\right)\left(u_{n}^{k+1}-u_{n}^{k}\right) \text { for } k \delta_{n} \leq t \leq(k+1) \delta_{n}, \\
& k=0, \ldots, n-1, \\
\bar{u}_{n}(t):= & u_{n}^{k+1} \text { for } k \delta_{n}<t \leq(k+1) \delta_{n}, k=0, \ldots, n-1, \bar{u}_{n}(0)=u_{n}^{0} .
\end{aligned}
$$

Then

$$
u_{n}(t)=u_{0}+\int_{0}^{t} \mathscr{G}\left(\bar{u}_{n}(\tau)\right) d \tau, \quad t \in[0, T],
$$

and with a suitable constant $S$ independent of $t \in[0, T]$ and $n \geq n_{0}$ :

$$
\left\|u_{n}(t)\right\|_{Y},\left\|\bar{u}_{n}(t)\right\|_{Y} \leq S .
$$

Consequently, $\left\|\mathscr{G}\left(\bar{u}_{n}(t)\right)\right\|_{Z}$ is bounded independently of $n$ and thus

$$
\left\|u_{n}(t)-u_{n}\left(t^{\prime}\right)\right\|_{Z} \leq L\left|t-t^{\prime}\right|
$$

with $L$ independent of $n$. Hence, the sequence $\left\{u_{n}\right\}$ is bounded and equicontinuous with values in $Z$, hence by Ascoli's theorem, we can assume without loss of generality

$$
u_{n} \rightarrow u \text { in } C([0, T], Z) .
$$


Moreover,

$$
u_{n}(t) \rightarrow u(t) \text { in } Y, \quad t \in[0, T] .
$$

To show this, fix $t \in[0, T]$ and choose an arbitrary subsequence $\left\{u_{n^{\prime}}(t)\right\}$. As it is bounded in $Y$, it has a weakly convergent subsequence $\left\{u_{n^{\prime \prime}}(t)\right\}$ for which $u_{n^{\prime \prime}}(t) \rightarrow u^{\star}$ in $Y$, hence also in $Z$, and thus $u^{\star}=u(t)$. Now (A.8) follows from a standard argument. An analogous argument shows

$$
u \in C_{w}([0, T], Y) .
$$

Furthermore, for $t \in\left(k \delta_{n},(k+1) \delta_{n}\right]$ we have

$$
\left\|\bar{u}_{n}(t)-u_{n}(t)\right\|_{Z}=\left\|u_{n}\left((k+1) \delta_{n}\right)-u_{n}(t)\right\|_{Z} \leq L \delta_{n},
$$

hence also

$$
\bar{u}_{n} \rightarrow u \text { in } C([0, T], Z),
$$

and, by the same arguments as for $u_{n}$ above,

$$
\bar{u}_{n}(t) \rightarrow u(t) \text { in } Y, \quad t \in[0, T] .
$$

As $\mathscr{G}$ is weakly sequentially continuous,

$$
\mathscr{G}\left(\bar{u}_{n}(t)\right) \rightarrow \mathscr{G}(u(t)) \text { in } Z, \quad t \in[0, T],
$$

and $\mathscr{G} \circ u \in C_{w}([0, T], Z)$. If $f$ is any bounded linear functional on $Z$, it follows that

$$
f\left(\int_{0}^{t} \mathscr{G}\left(\bar{u}_{n}(\tau)\right) d \tau\right)=\int_{0}^{t} f\left(\mathscr{G}\left(\bar{u}_{n}(\tau)\right)\right) d \tau \rightarrow \int_{0}^{t} f(\mathscr{G}(u(\tau))) d \tau, \quad n \rightarrow \infty,
$$

and hence

$$
f(u(t))=f\left(u_{0}\right)+\int_{0}^{t} f(\mathscr{G}(u(\tau))) d \tau, \quad t \in[0, T] .
$$

Consequently,

$$
f\left(\frac{u(t+h)-u(t)}{h}\right) \rightarrow f(\mathscr{G}(u(t))), \quad h \rightarrow 0,
$$

i.e.

$$
\frac{u(t+h)-u(t)}{h} \rightarrow \mathscr{G}(u(t)) \text { in } Z, \quad h \rightarrow 0 .
$$

Therefore $u \in C_{w}^{1}([0, T], Z)$ and $u$ satisfies (3.19). Finally, for $t \in\left(k \delta_{n},(k+1) \delta_{n}\right]$ we have

$$
\left\|\bar{u}_{n}(t)\left|\left\|^{2}=\right\| u_{n}^{k+1} \|\right|^{2} \leq \rho\left((k+1) \delta_{n}\right)+r_{n}\right.
$$

hence

$$
\left\|\bar{u}_{n}(t)\right\|^{2} \leq \rho\left(t+\delta_{n}\right)+r_{n} \text { for } 0 \leq t \leq T-\delta_{n} .
$$

Thus

$$
\|u(t)\|^{2} \leq \varliminf_{n \rightarrow \infty}\left\|\bar{u}_{n}(t)\right\|^{2} \leq \rho(t), \quad t \in[0, T] .
$$

For $t \rightarrow 0$ this implies, in particular,

$$
\varlimsup_{t \rightarrow 0}\|u(t)\|^{2} \leq \lim _{t \rightarrow 0} \rho(t)=\|u(0)\|\left\|^{2} \leq \varliminf_{t \rightarrow 0}\right\| u(t) \|^{2},
$$

hence $\|u(t)|\|\rightarrow|\|u(0)\||$ and consequently $u(t) \rightarrow u(0)$ in $Y$ as $t \rightarrow 0$. 


\section{References}

[1] Almgren, R.: Singularity formation in Hele-Shaw bubbles. Phys. Fluids 8 (1996), $344-352$.

[2] Beyer, K. \& Günther, M.: On the Cauchy problem for a capillary drop, PartI: Irrotational motion. Math. Meth. Appl. Sci. 21 (1998), 1149-1183.

[3] Beyer, K. \& Günther, M.: The Jacobi equation for irrotational free boundary flows. Analysis 20 (2000), 237-254.

[4] Constantin, P., Pugh, M.: Global solution for small data to the Hele-Shaw problem, Nonlinearity 6 (1993) 393-415

[5] Deckelnick, K. \& Elliott, C.: Local and global existence results for anisotropic Hele-Shaw flows. Proc. Roy. Soc. Edinburgh Sect. A 129 (1999), 265-294.

[6] Friedman, A., Reitich, F.: Nonlinear stability of a quasi-static Stefan problem with surface tension: a continuation approach. Ann. Scuola Norm. Sup. Pisa Cl. Sci.(4) 30 (2001) 341-403

[7] Giacomelli, L. \& Oтto, F.: Variational formulation for the lubrication approximation of the Hele-Shaw flow. Calc. Var. Part. Diff. Equations 3 (2001), 377-403.

[8] Gilbarg, D., Trudinger, N.S.: Elliptic partial differential equations of second order. Springer-Verlag, New York 1977.

[9] Günther, M. \& Prokert, G.: On Stokes Flow with Variable and Degenerate Surface Tension Coefficient. To appear in NoDEA.

[10] Hohlov, Yu.E. \& Reissig, M.: On classical solvability for the Hele-Shaw moving boundary problems with kinetic undercooling regularization. Euro. J. Applied Math. 6 (1995), 421-439.

[11] Hörmander, L.: The boundary problems of physical geodesy. Arch. Rat. Mech. Anal. 62 (1976), 1-52.

[12] Howison, S.D.: Complex variable methods in Hele-Shaw moving boundary problems. Euro. J. Applied Math. 3 (1992), 209-224.

[13] Kato, T. \& Lai, C. Y.: Nonlinear evolution equations and the Euler flow. $J$. Funct. Analysis 56 (1984), 15-28.

[14] Maruvada, S. R. K., Park, C. W. \& Yoon, D. Y.: The Influence of Surfactant on the Bubble Motion in Hele-Shaw Cells. Phys. Fluids 6 (1994), 3267-3275.

[15] Prokert, G.: Hyperbolic evolution equations for moving boundary problems. Euro. J. Applied Math. 10 (1999), 607-622.

[16] Prokert, G.: On Stokes flow driven by surface tension in the presence of a surfactant. Preprint RANA 03-21 TU Eindhoven, submitted.

[17] Romero, L.A.: The fingering problem in a Hele-Shaw cell. PhD Thesis (1981), California Institute of Technology.

[18] Saffman, P.G.: Viscous fingering in Hele-Shaw cells. J. Fluid Mech. 173 (1986), $73-94$. 
[19] Yi, Fahuai: Asymptotic behaviour of the solutions of the supercooled Stefan problem. Proc. Royal Soc. Edinburgh 127 A (1997), 181-190.

[20] Yu, Wanghui: A quasisteady Stefan problem with curvature correction and kinetic undercooling. J. Partial Diff. Eqs. 9 (1996), 55-70. 